\title{
A two-dimensional Stockwell transform for gravity wave analysis of AIRS measurements
}

\author{
Neil P. Hindley ${ }^{1}$, Nathan D. Smith ${ }^{1}$, Corwin J. Wright ${ }^{1}$, D. Andrew S. Rees ${ }^{2}$, and Nicholas J. Mitchell ${ }^{1}$ \\ ${ }^{1}$ Centre for Space, Atmosphere and Ocean Science, University of Bath, Bath, UK \\ ${ }^{2}$ Department of Mechanical Engineering, University of Bath, Bath, UK \\ Correspondence to: Neil P. Hindley (n.hindley@bath.ac.uk)
}

Received: 7 December 2015 - Published in Atmos. Meas. Tech. Discuss.: 18 January 2016

Revised: 5 May 2016 - Accepted: 6 May 2016 - Published: 9 June 2016

\begin{abstract}
Gravity waves (GWs) play a crucial role in the dynamics of the earth's atmosphere. These waves couple lower, middle and upper atmospheric layers by transporting and depositing energy and momentum from their sources to great heights. The accurate parameterisation of GW momentum flux is of key importance to general circulation models but requires accurate measurement of $\mathrm{GW}$ properties, which has proved challenging. For more than a decade, the nadir-viewing Atmospheric Infrared Sounder (AIRS) aboard NASA's Aqua satellite has made global, two-dimensional (2-D) measurements of stratospheric radiances in which GWs can be detected. However, one problem with current one-dimensional methods for GW analysis of these data is that they can introduce significant unwanted biases. Here, we present a new analysis method that resolves this problem. Our method uses a 2-D Stockwell transform (2DST) to measure GW amplitudes, horizontal wavelengths and directions of propagation using both the along-track and cross-track dimensions simultaneously. We first test our new method and demonstrate that it can accurately measure GW properties in a specified wave field. We then show that by using a new elliptical spectral window in the 2DST, in place of the traditional Gaussian, we can dramatically improve the recovery of wave amplitude over the standard approach. We then use our improved method to measure GW properties and momentum fluxes in AIRS measurements over two regions known to be intense hotspots of GW activity: (i) the Drake Passage/Antarctic Peninsula and (ii) the isolated mountainous island of South Georgia. The significance of our new 2DST method is that it provides more accurate, unbiased and better localised measurements of key GW properties compared to most current methods. The added flexibility offered by the
\end{abstract}

scaling parameter and our new spectral window presented here extend the usefulness of our 2DST method to other areas of geophysical data analysis and beyond.

\section{Introduction}

Gravity waves are a vital component of the atmospheric system. These propagating mesoscale disturbances can transport energy and momentum from their source regions to great heights. They thus are a key driving mechanism in the dynamics of the middle atmosphere through drag and diffusion processes (e.g. Fritts and Alexander, 2003, and references therein).

The accurate parameterisation of unresolved gravity waves in global climate models (GCMs) has proven to be a longstanding problem in the modelling community. One example of this is the "cold pole" bias (Butchart et al., 2011) suffered by nearly all GCMs, which has been suggested to be due to a deficiency of resolved and parameterised gravity wave drag near $60^{\circ} \mathrm{S}$. One reason for this is that these gravity wave parameterisations continue to be poorly constrained by observations (Alexander et al., 2010). The accurate measurement of gravity wave properties is thus critical for the development of the next and current generation of climate models. In the last decade, satellite-based remote-sensing has greatly increased our capability to make gravity wave observations on a global scale, but large discrepancies between observed and modelled fluxes still remain (Geller et al., 2013). This highlights the need for more accurate global and regional gravity wave measurements. 
The Atmospheric Infrared Sounder (AIRS) (Aumann et al., 2003) is a nadir-sounding spectral imager on board the Aqua satellite, launched in 2002. Part of the A-Train satellite constellation, AIRS scans the atmosphere over the range $\pm 49^{\circ}$ from the nadir of the satellite in a 90 pixel $(\sim 1800 \mathrm{~km})$ wide swath, using 2378 infrared channels along a sun-synchronous polar orbit. This continuous swath is archived in granules, usually 135 pixels $(\sim 2400 \mathrm{~km})$ alongtrack. Stratospheric gravity waves can be detected in these granules as radiance perturbations in the 15 and $4.3 \mu \mathrm{m} \mathrm{CO} 2$ emission bands (e.g. Alexander and Barnet, 2007; Hoffmann and Alexander, 2009; Hoffmann et al., 2013). AIRS measurements enable the study of stratospheric gravity waves at unprecedented horizontal resolution. In order to fully exploit these observations, accurate and easily reproducible analysis methods for the measurement of gravity wave properties must be developed.

The Stockwell transform (S-transform) (Stockwell et al., 1996; Stockwell, 1999) is a widely used spectral analysis technique for providing time-frequency (or distancewavenumber) localisation of a time series (or spatial profile). This capability makes the S-transform well suited to gravity wave analysis of a variety of geophysical data (e.g. Fritts et al., 1998; Alexander et al., 2008; McDonald, 2012; Wright and Gille, 2013). The S-transform has also been used in a variety of other fields, such as the planetary (Wright, 2012), engineering (Kuyuk, 2015) and medical sciences (Goodyear et al., 2004; Yan et al., 2015).

Alexander and Barnet (2007) developed a method for measuring gravity wave amplitudes, horizontal wavelengths and directions of propagation from AIRS granules using the one-dimensional (1-D) S-transform. In their method, the Stransform is computed for each cross-track row, and cospectra between adjacent cross-track rows are used to obtain spectral information in the along-track dimension. To find the dominant waves in each granule, these cross-track co-spectra are averaged together and up to five peaks are located in each averaged spectrum. The method of Alexander and Barnet (2007) provides good first-order measurement of the properties of the (up to five) dominant wave features in a granule, but it can introduce unwanted biases as discussed further in Sect. 5.

Here, we present a new analysis method. AIRS radiance measurements are two-dimensional (2-D) images; thus a gravity wave analysis method using a two-dimensional Stockwell transform (2DST) is a more logical approach. In this study we present a 2DST-based method for the measurement of gravity wave amplitudes, horizontal wavelengths and directions of propagation from AIRS measurements. Our method takes advantage of the spatial-spectral localisation capabilities of the S-transform in both dimensions simultaneously, equally and without bias.

South Georgia and the Antarctic Peninsula, together with the southern tip of South America, lie in a well-known hotspot of stratospheric gravity wave activity during austral winter, which has been extensively studied both observationally (Eckermann and Preusse, 1999; Jiang et al., 2002; Alexander and Teitelbaum, 2007; Baumgaertner and McDonald, 2007; Hertzog et al., 2008; Alexander et al., 2009; Alexander and Teitelbaum, 2011; Alexander and Grimsdell, 2013; Hindley et al., 2015) and with numerical modelling techniques (Hertzog et al., 2008; Plougonven et al., 2010; Shutts and Vosper, 2011; Hertzog et al., 2012; Sato et al., 2012; Plougonven et al., 2013) in the last decade. These mountainous regions are subjected to a strong wintertime circumpolar flow in the troposphere and stratosphere and, as a result, are major orographic gravity wave sources (e.g. Hoffmann et al., 2013). Despite this, discrepancies between observed and modelled gravity wave fluxes in this region are the largest anywhere on the planet (Geller et al., 2013). This unique geography of this important region provides a "natural laboratory" in which to make clear gravity wave measurements from space and is thus an ideal region in which to test our 2DST methodology on AIRS measurements.

In Sect. 2 we introduce AIRS data and the 1-D and 2D S-transforms. In Sect. 3 we apply the 2DST to a specified wave field, describing our methodology for spatial localisation of the dominant spectral components. In Sect. 4 we present a new alternative spectral window for use in the 2DST. In Sect. 5 we apply the 2DST to two selected AIRS granules measured over the Antarctic Peninsula/Drake Passage and South Georgia and discuss our results. Finally, in Sect. 6 we summarise the key results of this study and discuss the advantages of our 2DST method in the context of previous work.

\section{Data and spectral methods}

\subsection{AIRS data}

Gravity waves can be detected in AIRS radiance measurements as perturbations from a background state. Here, we use AIRS Level 1B radiance measurements from the $667.77 \mathrm{~cm}^{-1}$ channel. These Level 1 radiances have considerably higher horizontal resolution than operational Level 2 temperature retrievals due to retrieval choices imposed on the latter (Hoffmann and Alexander, 2009). We compute brightness temperature $T$ directly from radiance $R$ as

$$
\bar{T}=\frac{h c v}{k_{\mathrm{B}}}\left(\ln \left(\frac{2 h c^{2} v^{3}}{R}+1\right)\right)^{-1},
$$

where $h$ is Planck's constant, $c$ is the speed of light, $k_{\mathrm{B}}$ is Boltzmann's constant and $v=667.77 \mathrm{~cm}^{-1}$ is the wavenumber of the specified channel. Temperature perturbations $T^{\prime}$ from the local background state $\bar{T}$ are then extracted via a fourth-order polynomial fit (Wu, 2004; Alexander and Barnet, 2007). This fit removes limb-brightening and other largescale fluctuations. These brightness temperature perturbations are a more useful physical quantity with which to de- 
fine gravity wave amplitudes, since gravity wave energies and momentum fluxes are easier defined in terms of temperature perturbations (e.g. Ern et al., 2004). Common limitations in temperature perturbation-based momentum flux calculations, such as the assumption of a monochromatic wave and observational filtering effects, do remain, however, and are discussed in more detail in Sect. 5.3.

The weighting function of the $667.77 \mathrm{~cm}^{-1}$ channel peaks near $3 \mathrm{hPa}(\sim 40 \mathrm{~km})$, with a full width at half maximum of $\sim 12 \mathrm{~km}$ (Alexander and Barnet, 2007, also illustrated in Fig. 1 of Wright et al., 2016a). Gravity waves with vertical wavelengths shorter than $12 \mathrm{~km}$ are thus unlikely to be resolved and vertical wavelengths close to this limit will be strongly attenuated.

If the vertical wavelength is known, it is possible to correct for this attenuation by dividing the amplitude by an appropriate rescaling factor (Alexander and Barnet, 2007, their Fig. 4). Although methods for measuring long vertical wavelengths using multiple AIRS channels have been developed (e.g. Hoffmann and Alexander, 2009), we do not have direct measurements of vertical wavelength from our single AIRS channel, and so we do not apply such a correction to brightness temperature perturbations at this stage. The true amplitude of some waves in our initial analysis may therefore be between 2 and 5 times greater than the values shown. Later, however, for the estimation of momentum flux in Sect. 5.3, we do apply the attenuation correction described in Alexander and Barnet (2007).

\subsection{The Stockwell transform}

In its analytical form, the 1-D Stockwell transform (Stockwell et al., 1996) closely resembles a continuous wavelet transform (CWT) with a complex sinusoidal mother wavelet windowed with a scalable Gaussian window (Gibson et al., 2006). For time series data, this scalable Gaussian localises wave perturbations in the time domain through spectral localisation in the frequency domain.

For a smoothly varying, continuous and one-dimensional function of time $h(t)$, the generalised analytical form of the S-transform $S(\tau, f)$ (e.g. Pinnegar and Mansinha, 2003) is given as

$S(\tau, f)=\int_{-\infty}^{\infty} h(t) \omega_{\mathrm{g}}(t-\tau, f) e^{-i 2 \pi f t} \mathrm{~d} t$

where $\tau$ is translation in the time domain, $f$ is frequency and $\omega_{\mathrm{g}}(t-\tau, f)$ is a windowing function, scaled with frequency, that provides spatial and spectral localisation. Traditionally, $\omega_{\mathrm{g}}(t-\tau, f)$ takes the form of the normalised Gaussian window

$\omega_{\mathrm{g}}(t-\tau, f)=\frac{1}{\sigma \sqrt{2 \pi}} e^{\frac{-(t-\tau)^{2}}{2 \sigma^{2}}}$ where $\sigma$ is the standard deviation. A key aspect of the Gaussian window in Eq. (3) is that the standard deviation is scaled for each frequency as $\sigma=\frac{c}{|f|}$, where $c$ is a scaling parameter usually set to 1 (Mansinha et al., 1997a). This window is often referred to as the "voice Gaussian", which provides localisation of a specific frequency "voice" (Stockwell, 1999). Another key aspect of the Gaussian in Eq. (3) is the normalisation factor $1 / \sigma \sqrt{2 \pi}$, which ensures that the integral of the window over all $t$ is equal to unity, a requirement for any windowing function used in the S-transform. Substituting Eq. (3) into Eq. (2) allows us to write the S-transform more explicitly as

$S(\tau, f)=\frac{|f|}{c \sqrt{2 \pi}} \int_{-\infty}^{\infty} h(t) e^{-\frac{(t-\tau)^{2} f^{2}}{2 c^{2}}} e^{-i 2 \pi f t} \mathrm{~d} t$.

Typically, the scaling parameter $c$ is set to 1 (e.g. Stockwell et al., 1996; Alexander et al., 2008; Wright and Gille, 2013), but it may also be set to other values to achieve more specific time-frequency localisation requirements (e.g. Mansinha et al., 1997b; Fritts et al., 1998; Pinnegar and Mansinha, 2003). Setting $c>1$ provides enhanced frequency localisation at the expense of time localisation, and contrarily setting $c<1$ achieves enhanced time localisation at the expense of frequency localisation. This effect is discussed in more detail in Sect. 4.

To compute the S-transform using the form in Eq. (4), it seems we must compute a convolution involving the voice Gaussian and the time series for each frequency voice $f$, which can become quite computationally intensive. Stockwell (1999, their Sect. 3.5.1) showed that under the convolution theorem (Brigham, 1974), the time-domain convolution in Eq. (4) could be written as a frequency-domain multiplication as

$S(\tau, f)=\int_{-\infty}^{\infty} H(\alpha+f) e^{\frac{-2 \pi^{2} c^{2} \alpha^{2}}{f^{2}}} e^{i 2 \pi \alpha \tau} \mathrm{d} \alpha$,

where $H(\alpha+f)$ is a shifted version of $H(\alpha)$, which is in turn the frequency analogue of $H(t)$. The frequency-domain form of the voice Gaussian, denoted by $\omega_{\mathrm{g}}(\alpha, f)$, is given as

$\omega_{\mathrm{g}}(\alpha, f)=e^{\frac{-2 \pi^{2} c^{2} \alpha^{2}}{f^{2}}}$

The standard deviation $\sigma_{\alpha}$ of this frequency-domain Gaussian window in Eq. (6) scales with frequency as $\sigma_{\alpha}=|f| / c$. Note that this voice Gaussian is unnormalised; its peak value is equal to 1 in the frequency domain.

In this frequency-domain form, the S-transform is computed for each frequency voice $f$ as the inverse Fourier transform of the product of $H(\alpha+f)$ and the corresponding frequency-domain voice Gaussian $\omega_{\mathrm{g}}(\alpha, f)$ in Eq. (6). Crucially, writing the S-transform as the frequency-domain multiplication in Eq. (5) enables computationally efficient 
("fast") discrete Fourier transform (DFT) algorithms and simple multiplication operations to be used. The S-transform is most commonly implemented in this manner within the atmospheric sciences.

The S-transform has a number of desirable characteristics for geophysical data analysis. Unlike a CWT, the absolute magnitudes of the complex-valued S-transform coefficients in $S(\tau, f)$ are directly related to the true underlying amplitude of the corresponding frequency voice $f$ at each location $\tau$. Information regarding wave amplitude is not strictly recoverable from a CWT, since the corresponding CWT coefficients are pseudo-correlation coefficients between the signal and the analysing wavelet.

One disadvantage to using fast DFT algorithms in an Stransform implementation is the familiar coarse wavelength resolution at low frequencies, a limitation not encountered by the CWT. Since both the S-transform and DFT algorithms are easily extended to higher dimensions, however, the reduced computational expense of a DFT-based S-transform makes this a practical tool for large 2-D data sets. Retention of the wave amplitude information in the S-transform is another key advantage.

\subsection{The two-dimensional Stockwell transform}

The S-transform is easily extended to higher dimensions. For a two-dimensional image $h(x, y)$, the 2DST is given by (Mansinha et al., 1997a; Stockwell, 1999)

$$
\begin{aligned}
& S\left(\tau_{x}, \tau_{y}, f_{x}, f_{y}\right)=\int_{-\infty}^{\infty} \int_{-\infty}^{\infty} h(x, y) \\
& \times \frac{\left|f_{x}\right|\left|f_{y}\right|}{2 \pi c^{2}} e^{-\left(\frac{\left(x-\tau_{x}\right)^{2} f_{x}^{2}+\left(y-\tau_{y}\right)^{2} f_{y}^{2}}{2 c^{2}}\right)} e^{-i 2 \pi\left(f_{x} x+f_{y} y\right)} \mathrm{d} x \mathrm{~d} y,
\end{aligned}
$$

where $\tau_{x}, \tau_{y}$ are translation in the $x$ and $y$ directions respectively. Here, $f_{x}$ and $f_{y}$ are simple spatial frequencies (inverse of wavelength) in the $x$ and $y$ directions respectively, following the notation of Stockwell (1999). For the remainder of the present paper, however, we switch to using angular wavenumbers $k_{x}=2 \pi f_{x}$ and $k_{y}=2 \pi f_{y}$, since this notation is more commonly used in the atmospheric sciences. Rewriting Eq. (7) in terms of angular wavenumbers $k_{x}$ and $k_{y}$ gives

$$
\begin{aligned}
& S\left(\tau_{x}, \tau_{y}, k_{x}, k_{y}\right)=\int_{-\infty}^{\infty} \int_{-\infty}^{\infty} h(x, y) \\
& \times \frac{\left|k_{x}\right|\left|k_{y}\right|}{8 \pi^{3} c^{2}} e^{-\left(\frac{\left(x-\tau_{x}\right)^{2} k_{x}^{2}+\left(y-\tau_{y}\right)^{2} k_{y}^{2}}{8 \pi^{2} c^{2}}\right)} e^{-i\left(k_{x} x+k_{y} y\right)} \mathrm{d} x \mathrm{~d} y .
\end{aligned}
$$

The Gaussian windowing term in Eq. 8 describes the 2-D voice Gaussian $w_{g}\left(x-\tau_{x}, y-\tau_{y}, k_{x}, k_{y}\right)$, where

$$
w_{g}\left(x, y, k_{x}, k_{y}\right)=\frac{\left|k_{x}\right|\left|k_{y}\right|}{8 \pi^{3} c^{2}} e^{-\frac{k_{x}^{2} x^{2}+k_{y}^{2} y^{2}}{8 \pi^{2} c^{2}}} .
$$

This is the 2-D form of the 1-D Gaussian window in Eq. (3). Here, the standard deviations of the 2-D Gaussian window in Eq. (9) are scaled with wavenumber in the $x$ and $y$ directions as $2 \pi c /\left|k_{x}\right|$ and $2 \pi c /\left|k_{y}\right|$, where $c$ is a scaling parameter.

As discussed in Sect. 2.2, greater computational efficiency is achieved by computing the 2DST as an operation in the wavenumber domain as

$$
\begin{aligned}
& S\left(\tau_{x}, \tau_{y}, k_{x}, k_{y}\right)=\int_{-\infty}^{\infty} \int_{-\infty}^{\infty} H\left(\alpha_{x}+k_{x}, \alpha_{y}+k_{y}\right) \\
& \times \frac{1}{4 \pi^{2}} e^{-\left(\frac{2 \pi^{2} c^{2} \alpha_{x}^{2}}{k_{x}^{2}}+\frac{2 \pi^{2} c^{2} \alpha_{y}^{2}}{k_{y}^{2}}\right)} e^{i\left(\alpha_{x} \tau_{x}+\alpha_{y} \tau_{y}\right)} \mathrm{d} \alpha_{x} \mathrm{~d} \alpha_{y},
\end{aligned}
$$

where $H\left(\alpha_{x}+k_{x}, \alpha_{y}+k_{y}\right)$ is a shifted version of $H\left(\alpha_{x}, \alpha_{y}\right)$, which is in turn the wavenumber analogue of the input image $h(x, y)$. Here wavenumbers $k_{x}$ and $k_{y}$ are used to scale, in $\alpha_{x}$ and $\alpha_{y}$ directions respectively, the standard deviations of the wavenumber-domain form of the 2-D voice Gaussian $W_{\mathrm{g}}\left(\alpha_{x}, \alpha_{y}, k_{x}, k_{y}\right)$, which is given as

$W_{\mathrm{g}}\left(\alpha_{x}, \alpha_{y}, k_{x}, k_{y}\right)=e^{-\left(\frac{2 \pi^{2} c^{2} \alpha_{x}^{2}}{k_{x}^{2}}+\frac{2 \pi^{2} c^{2} \alpha_{y}^{2}}{k_{y}^{2}}\right)}$.

The 2DST is introduced and well described by Mansinha et al. (1997a) and Mansinha et al. (1997b), who demonstrated its promise for pattern analysis. It has since been discussed and applied in a variety of fields (Liu and Wong, 2007; Kocahan et al., 2008; Liu, 2009; Barry et al., 2012) but to our knowledge it has yet to be used for geophysical data analysis in the atmospheric sciences, despite the wide use of the 1-D form. In the following section, we describe our 2DST implementation methodology for the purpose of gravity wave analysis from 2-D data.

\section{2DST analysis of a specified wave field}

To assess the capabilities of the 2DST, it is logical to first apply it to a two-dimensional specified wave field containing synthetic waves with known characteristics.

We create a specified wave field $h(x, y)$ with dimensions $100 \times 100 \mathrm{~km}$ containing synthetic waves with unit amplitudes and known wavelengths. Wave amplitudes are defined as temperature perturbations $T^{\prime}$ in units of Kelvin. The synthetic waves are localised around their central locations with Gaussian functions (although note that they do overlap). We also add random ("salt and pepper") noise up to $10 \%$ of the wave amplitude.

We first compute the 2-D DFT $H\left(\alpha_{x}, \alpha_{y}\right)$ of our specified wave field $h(x, y)$. To recover an estimate of the underlying wave amplitude, we use the familiar symmetry around the zeroth frequency in the Fourier domain to recover a 2-D analogy of the analytic signal, following the approach of Stockwell (1999). A 2-D DFT contains four quadrants that 

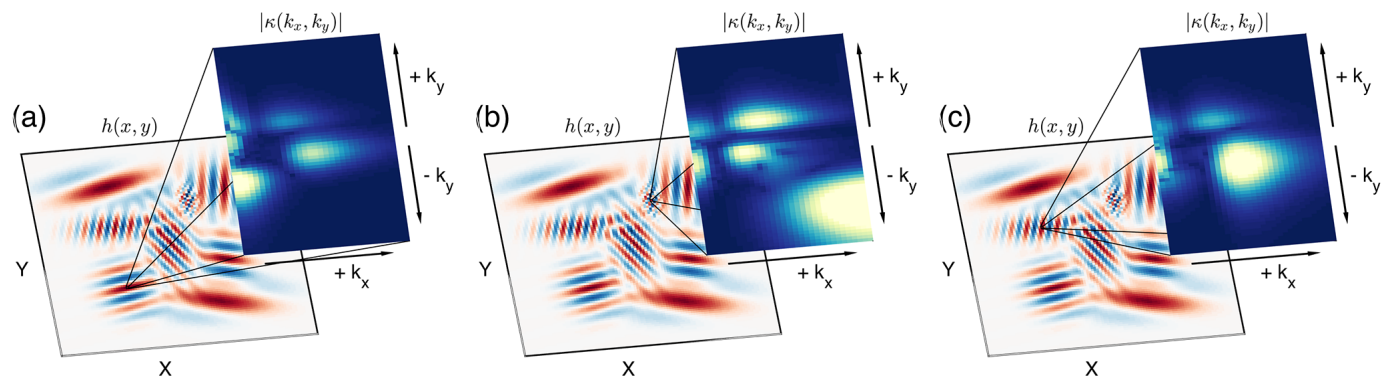

Figure 1. The specified wave field $h(x, y)$ (background) for which the two-dimensional Stockwell transform (2DST) has been computed. The absolute magnitudes of the localised 2DST wavenumber spectra $\left|\kappa\left(k_{x}, k_{y}\right)\right|$ (foreground) are plotted for three separate locations in (a), (b) and (c).

contain coefficients which are in complex-conjugate pairs with the coefficients in the opposite quadrant. The sum of these pairs always yields a real signal. By setting the coefficients of two of these quadrants to 0 , and doubling their opposite quadrants, we obtain a complex-valued image when we take the inverse DFT. The magnitude of this image is analogous to the underlying wave amplitude, while the complex part describes instantaneous phase. All coefficients not in a complex conjugate pair are unchanged. The full 2DST spectrum $S\left(\tau_{x}, \tau_{y}, k_{x}, k_{y}\right)$ can then be computed by taking the inverse 2-D DFT of the product of the shifted spectrum $H\left(\alpha_{x}-k_{x}, \alpha_{y}-k_{y}\right)$ and the corresponding voice Gaussian $W_{\mathrm{g}}\left(\alpha_{x}, \alpha_{y}, k_{x}, k_{y}\right)$ for each wavenumber voice $k_{x}$ and $k_{y}$.

For our purposes, we do not need to evaluate $S\left(\tau_{x}, \tau_{y}, k_{x}, k_{y}\right)$ for all positive and negative wavenumbers, since evaluating all positive and negative values of $k_{y}$ and only the positive values of $k_{x}$ gives us all the degrees of freedom. There is a residual $180^{\circ}$ ambiguity in wave propagation direction which cannot be broken without additional information, which is supplied in Sect. 5.3.

A useful aspect of our implementation is that, like the CWT, we can compute the 2DST for any individual or range of permitted wavenumber voices by applying the appropriate wavenumber-scaled Gaussian windows. Although the permitted wavenumber voices in the spectral domain are evenly spaced, their corresponding wavelengths are limited to integer fractions (i.e. $1 / n$ where $n=0,1,2, \ldots, N-1$ ) of the number of elements $N$ in each dimension. This is an unavoidable consequence of using computationally efficient DFT algorithms, which results in the familiar coarse spectral resolution seen at long wavelengths.

The ability to analyse an image at specific wavenumbers is a desirable aspect in geophysical data analysis, where some a priori information regarding the spectral range of wavenumbers detectable in a given data set can be used to reduce the impact of unphysical, spurious or noisy results in 2DST analysis.

\subsection{Measuring gravity wave properties}

The 2DST $S\left(\tau_{x}, \tau_{y}, k_{x}, k_{y}\right)$ of our specified wave field is a four-dimensional (4-D) object. For each location in $h(x, y)$, a two-dimensional complex-valued image of the localised spectral coefficients $\kappa\left(k_{x}, k_{y}\right)$ is evaluated.

Figure 1 shows a specified wave field $h(x, y)$ for which the 2DST has been computed. The absolute magnitude of the localised two-dimensional wavenumber spectrum $\left|\kappa\left(k_{x}, k_{y}\right)\right|$ is plotted for three different example locations. The coefficients of $\left|\kappa\left(k_{x}, k_{y}\right)\right|$ can be directly interpreted as the underlying amplitudes of waves with wavenumbers $k_{x}$ and $k_{y}$ at a given location in the specified wave field. As discussed in previous studies (e.g. Wright and Gille, 2013; Wright et al., $2015)$, there are likely to be multiple peaks in $\left|\kappa\left(k_{x}, k_{y}\right)\right|$ corresponding to overlapping waves at the same location in $h(x, y)$. Indeed, in Fig. 1b we examine a location in the specified wave field where a small, high-wavenumber wave is located at the intersection of four lower-wavenumber waves. The localised spectrum computed by the 2DST shown in the foreground represents this feature well. The maximum spectral response is located in a peak at high $k_{x}$ and $k_{y}$ wavenumbers, with four smaller spectral peaks at lower wavenumbers with lower spectral responses.

A 4-D complex-valued function can be difficult to visualise. A more useful product might be a series of twodimensional images, the same size as the input image, that contain the characteristics of the dominant wave at each location. In the implementation presented here, we neglect overlapping waves and identify a single dominant wave for each location in $h(x, y)$

For each such location, we record the complex coefficient of $\kappa\left(k_{x}, k_{y}\right)$ located at the spectral peak of $\left|\kappa\left(k_{x}, k_{y}\right)\right|$. This yields one complex-valued image $\xi\left(\tau_{x}, \tau_{y}\right)$, with the same dimensions as the specified wave field $h(x, y)$, which contains the amplitude and phase of the dominant wave at each location.

The location of the spectral peak in $\left|\kappa\left(k_{x}, k_{y}\right)\right|$ also gives us the wavenumbers $k_{x}$ and $k_{y}$ to which this peak coefficient corresponds. Hence, we can produce two further im- 

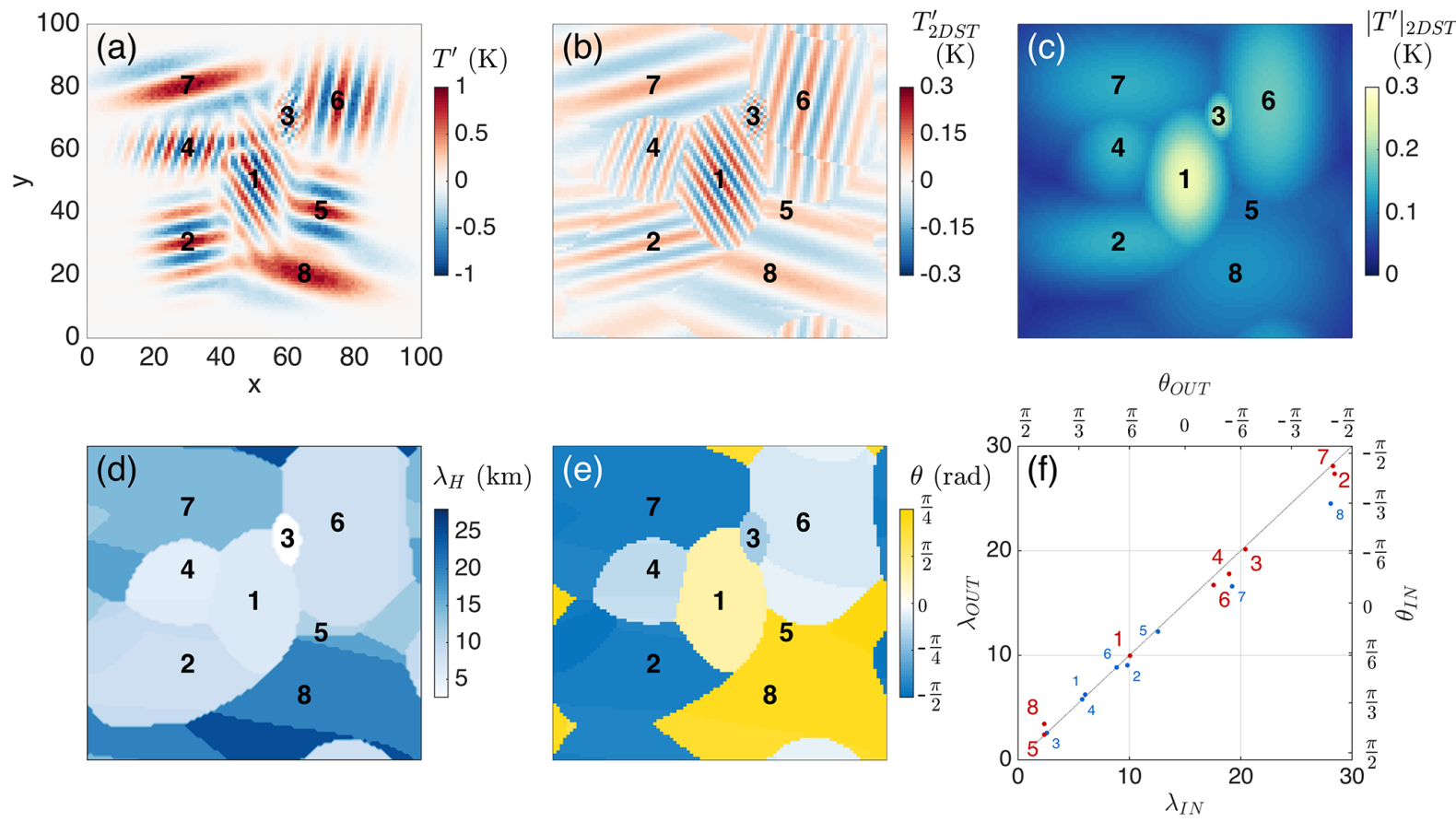

Figure 2. The specified wave field (a), containing synthetic waves numbered 1-8, for which our two-dimensional Stockwell transform analysis has been performed. The "reconstructed" wave field $T^{\prime}{ }_{2-\mathrm{DST}}$, underlying wave amplitudes $\left|T^{\prime}\right|_{2-\mathrm{DST}}$, horizontal wavelengths $\lambda_{\mathrm{H}}$ and directions $\theta$ (measured anticlockwise from the positive $x$ direction) are shown in (b), (c), (d) and (e) respectively. Distances and wavelengths have units of kilometres and amplitudes have units of Kelvin. Panel (f) compares input and measured wavelengths (blue numbered dots) and input and measured propagation angles (red numbered dots) for the eight synthetic waves. The dashed grey line in (f) shows $1: 1$ correspondence.

ages $K_{x}\left(\tau_{x}, \tau_{y}\right)$ and $K_{y}\left(\tau_{x}, \tau_{y}\right)$ which contain the dominant wavenumbers at each location in the specified wave field to which the coefficients of $\xi\left(\tau_{x}, \tau_{y}\right)$ correspond.

Thus, in the three images $\xi\left(\tau_{x}, \tau_{y}\right), K_{x}\left(\tau_{x}, \tau_{y}\right)$ and $K_{y}\left(\tau_{x}, \tau_{y}\right)$, we can measure the amplitudes, phases, wavelengths and propagation directions of the dominant wave features at each location in our specified wave field.

Figure 2a shows our specified wave field $h(x, y)$. The central locations of the eight synthetic waves with unit amplitudes and known wavelengths are numbered 1-8.

By taking the real part of the complex-valued image $\xi\left(\tau_{x}, \tau_{y}\right)$ containing the dominant coefficients, we can recover a "reconstruction" of the specified wave field, which is shown in Fig. 2b. This is made possible by the approach described above in Sect. 3.1.

The 2DST identifies the different spectral regimes of the specified wave field very well, but the reconstructed wave amplitudes are reduced by comparison to their original values.

We suspect the main reason for the reduced amplitudes relates to the "spreading" of spectral power in the transform. Here, as is often the case for gravity waves in the real world, our simulated waves form small wave packets, where wave amplitude decreases around a central location. Such wave packets are usually represented in the spectral domain as some combination of wavenumber voices, in addition to the dominant wavenumber of each of the packets, in order to accurately describe their spatial properties. This means that the spectral power of a single, non-infinite wave packet can be spread across multiple wavenumber voices. Spectral leakage can further contribute to this effect.

The Gaussian window in the 2DST is equal to 1 at its central location but immediately falls away with increasing radius. This means that any spectral power contained in adjacent wavenumber voices, which is required to fully reconstruct the wave, is reduced. When the inverse DFT is computed, the recovered wave amplitude at this location is thus often diminished.

A further reason for the diminished amplitude recovery in Fig. $2 b$ is due to wave undersampling. This undersampling effect is worse for longer wavelengths, since fewer wave cycles are present in the same-sized region of the image. The wave undersampling limitations of the S-transform are well understood in one dimension (Wright, 2010; Wright et al., 2015).

Figure $2 \mathrm{c}$ shows the absolute magnitude of the complexvalued image $\left|\xi\left(\tau_{x}, \tau_{y}\right)\right|$, which corresponds to the full underlying amplitude of the dominant wave at each location. This output is useful for defining regions of the specified 
wave field that do or do not contain clear and obvious wave features (McDonald, 2012).

The horizontal wavelength $\lambda_{H}\left(\tau_{x}, \tau_{y}\right)=\left(K_{x}\left(\tau_{x}, \tau_{y}\right)^{2}+\right.$ $\left.K_{y}\left(\tau_{x}, \tau_{y}\right)^{2}\right)^{-1 / 2}$ of the dominant wave at each location is shown in Fig. 2d. Again, the different regimes of each wave in the specified wave field are clearly distinguished.

The direction of wave propagation $\theta\left(\tau_{x}, \tau_{y}\right)$, measured anticlockwise from the $x$ axis, is found as $\tan ^{-1} \frac{K_{x}}{K_{y}}$ and plotted as Fig. 2e. Note that $\theta\left(\tau_{x}, \tau_{y}\right)$ is subject to a $\pm \pi$ radian ambiguity, which is reconciled with a priori information in our AIRS analysis in Sect. 5.3.

To assess the effectiveness of our spectral analysis of the specified wave field, we compare the known wavelengths and propagation angles of the synthetic waves in the test image with the 2DST-measured wavelengths and propagation angles in Fig. 2f. For each wave numbered 1-8, blue dots show the input wavelength $\lambda_{\text {IN }}$ against measured wavelength $\lambda_{\text {OUT }}$, indicating that the 2DST measures the horizontal wavelengths and propagation angles in the test image very well. Generally, shorter wavelengths are well resolved but longer wavelengths are slightly underestimated. This may be due to the coarse spectral resolution of DFT-based methods for waves with wavelengths that are a large fraction of the image size, since such waves can be more susceptible to spectral leakage problems.

\section{An alternative spectral window}

The use of the Gaussian window in the S-transform has some convenient mathematical advantages; it is analytically simple and has a definite integral over an infinite range. However, when it is used for 2DST analysis an unfortunate side effect of the Gaussian window is the poor recovery of wave amplitude, discussed in the previous section. Although a Gaussian is traditionally used, any suitable apodizing function may be used, so long as its spatial integral is equal to unity (Stockwell, 2007). For example, Pinnegar and Mansinha (2003) used an asymmetric hyperbolic time-domain window for enhanced measurement of the onset times of one-dimensional time series components.

In this section, we introduce a new spectral windowing function for the 2DST. This new function takes the shape of an ellipse in the wavenumber domain, and a first-order Bessel function of the first kind $\mathbf{J}_{1}(z)$ function with a scaled $1 / z$ envelope in the spatial domain (for definition of $z$ see Eq. (14) below). For this reason we refer to this window as the Elliptic-Bessel window. We find that when AIRS measurements are analysed with the 2DST using this new Elliptic-Bessel window in place of the traditional Gaussian, the measurement of gravity wave amplitudes is greatly improved. Spectral resolution is also improved slightly, without adversely compromising spatial resolution.

\subsection{The Elliptic-Bessel window}

As discussed in Sect. 3.1, the spectral peaks in a DFT spectrum have a characteristic width, where the spectral power is spread in a broad peak around the central wavenumber. This spectral power is slightly reduced when a Gaussian window is applied, due to the immediate decrease in the Gaussian function around the central location. The effect can be mitigated, but not fully reconciled, by decreasing the scaling parameter $c$, which broadens the Gaussian window in the wavenumber domain. However, this decreases the width of the spatial window, which increases the effect of wave undersampling for low wavenumbers.

One solution to this problem is to use a window that is an ellipse in the wavenumber domain. Here we introduce an Elliptic-Bessel window $W_{\text {eb }}$, defined in the wavenumber domain as the ellipse

$W_{\mathrm{eb}}\left(\alpha_{x}, \alpha_{y}, k_{x}, k_{y}\right)=\left\{\begin{array}{l}0 \text { for }\left(\frac{\alpha_{x}}{a}\right)^{2}+\left(\frac{\alpha_{y}}{b}\right)^{2} \geqslant 1 \\ 1 \text { for }\left(\frac{\alpha_{x}}{a}\right)^{2}+\left(\frac{\alpha_{y}}{b}\right)^{2}<1\end{array}\right.$,

where $a=\left|k_{x}\right| / 2 \pi c$ and $b=\left|k_{y}\right| / 2 \pi c$ are the widths in the $\alpha_{x}$ and $\alpha_{y}$ directions. We see that the semi-major and semi-minor axes of this "voice ellipse" scale with angular wavenumbers and are equal to the standard deviations of the equivalent voice Gaussian window in Eq. (11).

A key feature of this new window is that, in the wavenumber domain, it does not immediately decrease with displacement from the central location but rather has a scalable elliptical region within which the function is equal to unity. Thus, the window captures a much greater extent of the targeted spectral peak at $k_{x}$ and $k_{y}$, which can greatly improve wave amplitude recovery compared to the traditional Gaussian window. The width of $W_{\text {eb }}$ can also be more carefully adjusted using the scaling parameter $c$. This window can then be used in place of the Gaussian windowing term in Eq. (10).

One requirement for any apodizing window used in the Stockwell transform is that the spatial integral of the function must be equal to unity. This is so that the spatial integral of the Stockwell transform is equal to the Fourier transform $H\left(k_{x}, k_{y}\right)$ (Mansinha et al., 1997a), namely

$\int_{-\infty}^{\infty} \int_{-\infty}^{\infty} S\left(\tau_{x}, \tau_{y}, k_{x}, k_{y}\right) \mathrm{d} \tau_{x} \mathrm{~d} \tau_{y}=H\left(k_{x}, k_{y}\right)$,

which has the useful result of making the 2DST fully invertible (Stockwell, 1999, 2007).

The normalisation term $\left|k_{x}\right|\left|k_{y}\right| / 8 \pi^{3} c^{2}$ in the traditionally used Gaussian window in Eq. (9) ensures that the Gaussian window satisfies this requirement. To check that the EllipticBessel function is admissible as an apodizing function, we must first find its spatial form, then check that its spatial integral is also equal to unity. 

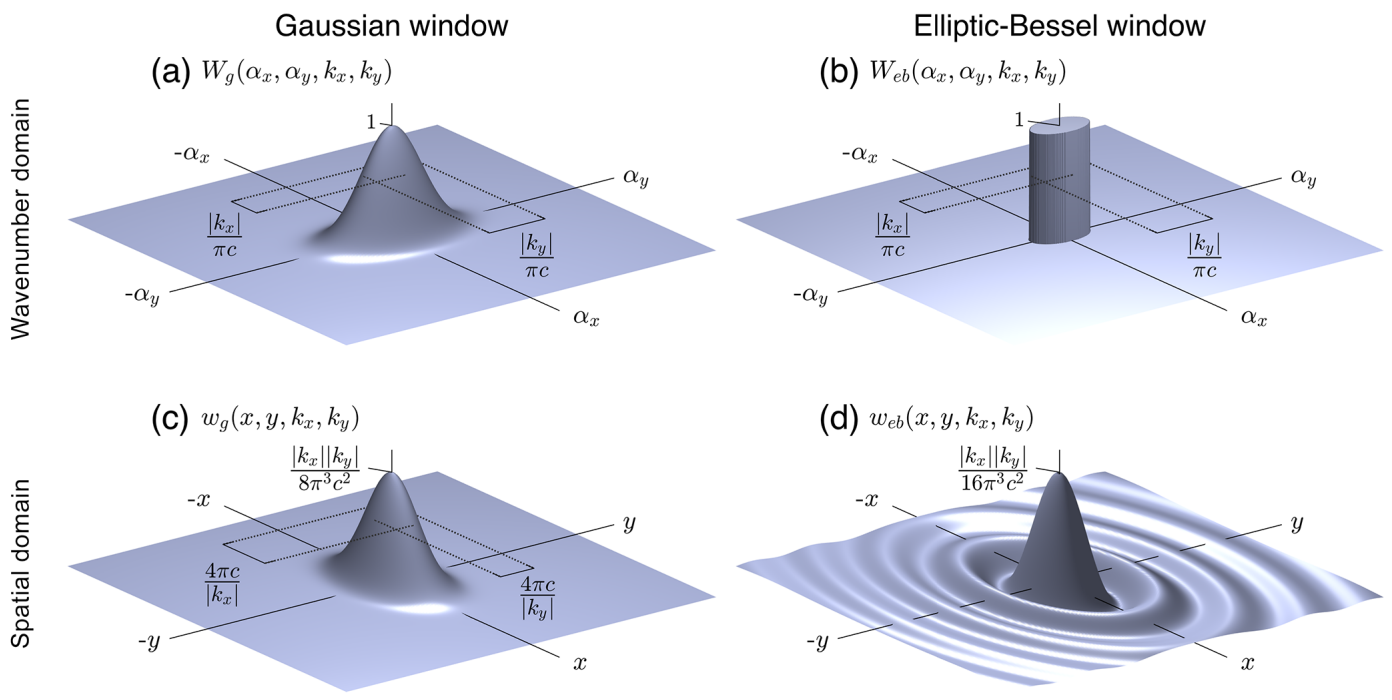

Figure 3. Illustrative surface plots of the wavenumber-domain (top row) and spatial-domain (bottom row) forms of the traditional Gaussian $(\mathbf{a}, \mathbf{c})$ and new Elliptic-Bessel $(\mathbf{b}, \mathbf{d})$ windowing functions used in the two-dimensional Stockwell transform for arbitrary wavenumbers $k_{x}$ and $k_{y}$ and scaling parameter $c$. The semi-major and semi-minor axes of the Elliptic-Bessel window in (b) are equal to the corresponding standard deviations of the Gaussian window in (a), where both windows have a central value equal to unity. In the spatial domain, the Elliptic-Bessel window in (d) has a central value equal to half that of the Gaussian window in (c), both of which have spatial integrals equal to unity. For details, see text.

The Elliptic-Bessel window $W_{\mathrm{eb}}\left(\alpha_{x}, \alpha_{y}, k_{x}, k_{y}\right)$ is easily defined in the wavenumber domain as an ellipse, but its spatial form, which we denote as $w_{\mathrm{eb}}\left(x, y, k_{x}, k_{y}\right)$, is given as

$w_{\mathrm{eb}}\left(x, y, k_{x}, k_{y}\right)=\frac{\left|k_{x}\right|\left|k_{y}\right|}{8 \pi^{3} c^{2}} \frac{\mathbf{J}_{1}(z)}{z}$,

where $\mathbf{J}_{1}$ is the first-order Bessel function of the first kind (Abramowitz and Stegun, 1964) and

$z=\frac{1}{2 \pi c} \sqrt{k_{x}^{2} x^{2}+k_{y}^{2} y^{2}}$.

A short derivation of the function in Eq. (14) is provided in Appendix A1. Fortunately, the spatial integral of Eq. (14) is indeed equal to unity, proof of which is presented in Appendix A2. This confirms that the Elliptic-Bessel window is admissible as an apodizing function in the 2DST and validates its use in this study and beyond.

To recap our notation in this study, we have described two windowing functions for the 2DST: the traditional Gaussian and the new Elliptic-Bessel windows, which we denote in the spatial domain as $w_{\mathrm{g}}\left(x, y, k_{x}, k_{y}\right)$ and $w_{\mathrm{eb}}\left(x, y, k_{x}, k_{y}\right)$ respectively and in the wavenumber domain as $W_{\mathrm{g}}\left(\alpha_{x}, \alpha_{y}, k_{x}, k_{y}\right)$ and $W_{\mathrm{eb}}\left(\alpha_{x}, \alpha_{y}, k_{x}, k_{y}\right)$ respectively, where the $W_{\mathrm{g}}$ and $W_{\mathrm{eb}}$ are the Fourier transforms of $w_{\mathrm{g}}$ and $w_{\mathrm{eb}}$.

Figure 3 shows three-dimensional surface plots of the spatial and wavenumber domain forms of the traditional 2-D Gaussian and new Elliptic-Bessel windows used in the 2DST here.
The surfaces in Fig. 3a and c show the wavenumberdomain and spatial-domain forms of the Gaussian window, for arbitrary wavenumbers $k_{x}$ and $k_{y}$. As discussed above, the maximum value of the Gaussian is equal to unity in the wavenumber domain but equal to $\left|k_{x}\right|\left|k_{y}\right| / 8 \pi^{3} c^{2}$ in the spatial domain such that its spatial integral is equal to unity. This is a requirement of any windowing function in the Stransform. The standard deviations of the $w_{\mathrm{g}}$ and $W_{\mathrm{g}}$ scale with wavenumbers $k_{x}$ and $k_{y}$ as described in Sect. 2.3 (Eqs. 9 and 11), providing the voice Gaussian.

Likewise, Fig. $3 \mathrm{~b}$ and d show the wavenumber-domain $\left(W_{\mathrm{eb}}\right)$ and spatial-domain $\left(w_{\mathrm{eb}}\right)$ forms of the Elliptic-Bessel window, for the same arbitrary wavenumbers $k_{x}$ and $k_{y}$ as used for the Gaussian windows in panels a and c. The semimajor and semi-minor axes of the elliptic region in Fig. $3 b$ are scaled with wavenumbers $k_{x}$ and $k_{y}$ and are equal to the standard deviations of the equivalent Gaussian in Fig. 3a, providing the voice ellipse.

The spatial-domain form of the Elliptic-Bessel window $w_{\mathrm{eb}}$, described by a Bessel-shaped function within an envelope, is shown in Fig. 3d and described by Eq. (14). The maximum value of $w_{\mathrm{eb}}$ is $\left|k_{x}\right|\left|k_{y}\right| / 16 \pi^{3} c^{2}$, which ensures that its spatial integral is equal to unity. This is equal to half of the maximum value of the equivalent Gaussian in Fig. 3c, since the terms involving $z$ in Eq. (14) tend to $1 / 2$ as $x \rightarrow 0$ and $y \rightarrow 0$. The width of the central region of $w_{\mathrm{eb}}$ is very slightly wider than the equivalent Gaussian, resulting in slightly coarser spatial resolution. However, the ability to 
(a)

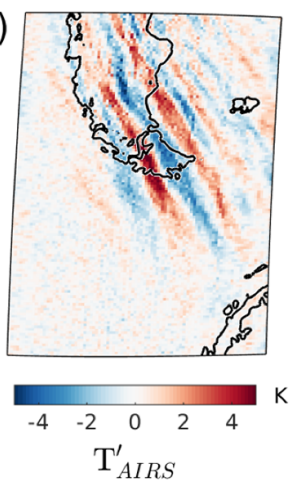

Gaussian, $\mathrm{c}=1$

(b)

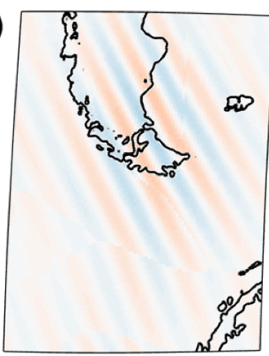

(e)

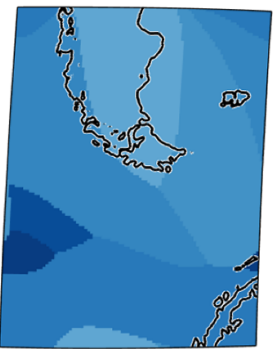

Gaussian, $\mathrm{c}=0.25$

(c)

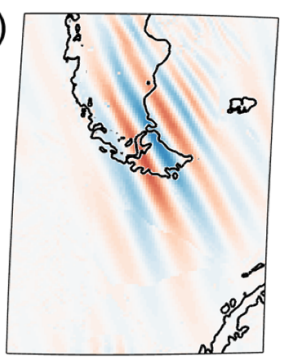

(f)

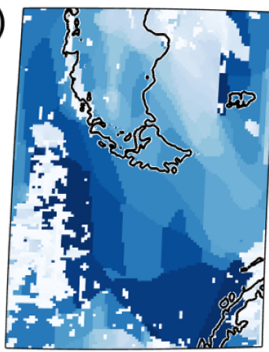

Elliptic-Bessel, $\mathrm{c}=0.25$

(d)

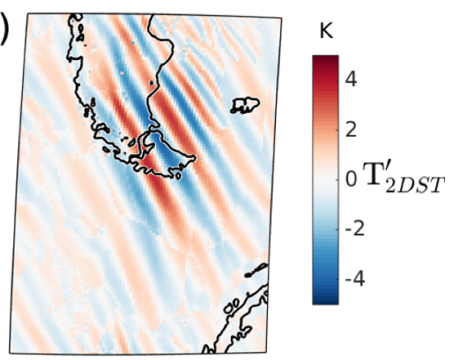

(g)

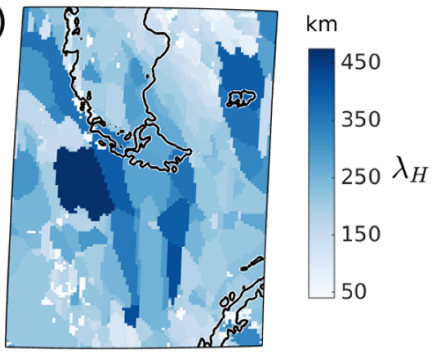

Figure 4. Orthographic projection of AIRS brightness temperature perturbations $T_{\mathrm{AIRS}}^{\prime}$ (a) from a granule over the Southern Andes at 05:30 UTC on 24 May 2008, with "reconstructed" temperature perturbations $T_{2-D S T}^{\prime}$ and horizontal wavelengths $\lambda_{\mathrm{H}}$ computed using the 2DST with three different windowing approaches: $(\mathbf{b}, \mathbf{e})$ a Gaussian window with scaling parameter $c=1,(\mathbf{c}, \mathbf{f})$ a Gaussian window with $c=0.25$ and $(\mathbf{d}, \mathbf{g})$ the Elliptic-Bessel window with $c=0.25$.

"tune" the 2DST with the scaling parameter $c$ ensures that this effect can be compensated by a reasonable trade-off.

In the next section we show that the use of the EllipticBessel window in the 2DST, in place of the traditional Gaussian window, significantly improves wave amplitude recovery. This is very useful for our analysis of AIRS data in Sect. 5.

\subsection{Invertibility}

A very convenient aspect of the S-transform is its invertibility. Since we have shown here that both the traditional Gaussian and new Elliptic-Bessel windows have spatial integrals equal to unity, the 2DST can be completely inverted to recover the original 2-D image, whichever of these windows or real non-zero positive values of the scaling parameter $c$ are used. Note that a traditional 1-D or 2-D CWT does not necessarily have this capability. The fact that we can achieve such flexibility in spatial-spectral resolutions by swapping windows or by adjusting $c$, yet still retain the capability of inversion, further highlights the strength of the 2DST as a tool for spatial-spectral analysis of geophysical data.

Unfortunately, to take full advantage of DFT algorithms and the inversion capability of the 2DST for AIRS data, we must compute the 2DST using all permitted wavenumber voices in both dimensions. This requires nearly 12000 inverse DFT calculations for each AIRS granule using the traditional voice-by-voice implementation described here, the computational load of which could be quite impractical for large-scale studies. Interpolating AIRS measurements to a coarser resolution with fewer pixels could be one solution to reduce computational cost, but this will obviously undersample short horizontal wavelengths in the data. Faster methods for computing the S-transform have been developed (Brown et al., 2010) which may increase practicality in the future. Other steps, such as avoiding programming loops and ensuring that any 2-D objects to be transformed have dimensions that are powers of two, may also reduce relative computational expense.

\subsection{The effect of window choices on AIRS analysis}

Figure 4 shows an AIRS granule over the Southern Andes measured on 24 May 2008, analysed using the 2DST with three different windowing approaches.

In Fig. $4 \mathrm{~b}$ and e, we use a Gaussian windowing function with the scaling parameter $c$ set to 1 . This is the window usually used in 1DST implementations. We see that, as discussed above, this choice of window is only able to recover the very general, long-horizontal wavelength features of the granule, with poor spatial localisation and significantly reduced amplitude. This is due to a large proportion of the spectral response being lost by the windowing Gaussian when applied to two dimensions.

We can reduce the impact of this by decreasing the scaling parameter $c$, which broadens (narrows) the spectral (spatial) window. This provides improved amplitude recovery and improved spatial localisation at the expense of spectral localisa- 
tion (Fritts et al., 1998). Since we only select a single dominant spectral peak for each location on the granule, this is acceptable for our purposes. The "reconstructed" perturbations and horizontal wavelengths (Fig. $4 \mathrm{c}$ and $\mathrm{f}$ ) are now much more representative of the wave features in the granule.

One problem remains, however. By decreasing $c$, we narrow our spatial window. In regions where wave amplitudes are low, such as the bottom-left corner of Fig. 4a, this narrow Gaussian window starts to undersample long wavelengths, such that only very short wavelengths are attributed to the region. The Elliptic-Bessel window used in Fig. $4 \mathrm{~d}$ and g performs better at recovering the underlying larger-scale structure of the granule, without defaulting to the small-scale noisy variations. Amplitude recovery at all wavelengths is also improved over either of the Gaussian approaches.

In the general case, these low-amplitude, small-scale variations are unlikely to be due to gravity waves with vertical wavelengths visible to AIRS, so their recovery is something we try to avoid. Furthermore, such wavelengths are very close to or at the Nyquist limit for these data. Our confidence in their measurement is thus very low, yet the momentum fluxes they transport can dominate. We discuss this further in Sect. 5.4.

For the windowing functions considered, it is clear from Fig. 4 that the scaling parameter $c$ has a significant effect in determining the spatial-spectral localisation capabilities of the 2DST. The Elliptic-Bessel windowing function, with a scaling parameter of $c=0.25$, was selected for our AIRS analysis in the next section. This choice provided the best trade-off between spatial and spectral localisation of different wave regimes in AIRS measurements.

As discussed in Sect. 3.1, the 2-D images $\xi\left(\tau_{x}, \tau_{y}\right)$, $K_{x}\left(\tau_{x}, \tau_{y}\right)$ and $K_{y}\left(\tau_{x}, \tau_{y}\right)$ contain the dominant measured wave amplitudes and wavelengths at each location on the granule. These images are computed on a pixel-by-pixel basis, selecting a single monochromatic wave with the largest amplitude in the localised spectrum for each pixel.

As a result, the reconstructed images shown in Fig. 4b$\mathrm{d}$, computed by taking the real part of the complex image $\xi\left(\tau_{x}, \tau_{y}\right)$, will never be perfect representations of the input data, but they will provide a "best guess" of the dominant features of the granule.

Since we have shown that the 2DST is fully invertible for both the Gaussian and Elliptic-Bessel windowing approaches (Sect. 4 and Appendix A), a complete reconstruction of the input image is of course producible by taking the "inverse" of the full 4-D 2DST object, but here we desire 2-D "maps" of wave properties, so a best guess method is used.

A possible quantitative metric to assess the first-order effectiveness of our 2DST analysis in Fig. 4 could be to compare the variance of the input image with the variances of each of the reconstructions. However, since the reconstructions are computed as a best guess method on a pixel-bypixel basis, their total variance is not readily related to the total variance of the input image and thus may not be mean- ingful as a comparison. Furthermore, such use of the image variance would only be appropriate if the distribution of perturbations was unimodal and ideally Gaussian, which is not the case for an image of a sinusoidal wave. In practice, however, we generally expect the variance of the reconstruction not to exceed the variance of the input image, since wave amplitudes computed on a pixel-by-pixel basis from a localised spectrum will usually be underestimated for the reasons given in Sect. 3.1.

It is not impossible that in some rare cases the total variance of the reconstruction could exceed the total variance of the input image, for example due to the spatial extent of a wave feature being slightly overestimated. If the localised spectrum for one pixel is affected a larger-amplitude wave feature in one of its neighbouring pixels, this can result in subtle artificial "borders" between different wave regimes in the reconstructions. This is not a limitation of the 2DST itself, but arises in the somewhat forced extraction of localised gravity wave parameters contained in the 4-D Stockwell transform object $S\left(\tau_{x}, \tau_{y}, k_{x}, k_{y}\right)$ in order to produce the 2-D image. This effect should be carefully considered in future work to ensure wave properties are not over-represented.

\section{AIRS gravity wave analysis using the 2DST}

In this section, we use our 2DST-based method to perform gravity wave analysis on two-dimensional granules of AIRS radiance measurements, comparing our analysis to that of previous studies. We use the 2DST to measure gravity wave amplitudes, horizontal wavelengths, and directions of propagation. We then use ECMWF-derived wind speeds and the assumption of an orographic wave source to infer vertical wavelengths and make estimates of gravity wave momentum flux (the vertical flux of horizontal pseudomomentum) by closely following the method of Alexander et al. (2009).

\subsection{AIRS granule selection and pre-processing}

The first AIRS granule selected for our study is granule 32 of 6 September 2003, over South Georgia. The second granule is a 135-pixel swath over the intersection between granules 39 and 40 on 2 August 2010, located over the Antarctic Peninsula and Drake Passage.

Alexander et al. (2009) and Hoffmann et al. (2014) performed an analysis of these AIRS granules over South Georgia and the Antarctic Peninsula respectively. Their studies measured wave amplitudes, horizontal wavelengths and wave propagation directions using a 1-D S-transform method, as described by Alexander and Barnet (2007). In their method, the 1-D S-transform is computed for each cross-track row. Then, covariance spectra are computed between pairs of adjacent cross-track rows to measure phase shifts in the along-track direction, from which along-track wavelengths can be inferred. To find the dominant wave fea- 
tures in a granule, these co-spectra are averaged together and up to five spectral peaks are found in this averaged spectrum. This approach can provide computationally fast, first-order gravity wave analysis of AIRS granules, and it has been used in numerous other studies (e.g. Alexander and Teitelbaum, 2011; Alexander and Grimsdell, 2013; Wright et al., 2016a).

One limitation of this method is that the phase difference measurements required to recover along-track wavenumbers can introduce a strong cross-track bias in resolved features, since the S-transform is only computed in the cross-track direction. In addition, waves which occupy only small regions of the granule in the along-track direction may also be underrepresented in the averaged co-spectrum. Furthermore, selecting no more than five dominant waves in the averaged cospectrum implicitly limits the maximum number of available along-track wavenumber voices to no more than five for each location on the entire granule. The use of a 2DST is a logical solution to each of these problems. With the increased convenience of computational power since the study of Alexander and Barnet (2007), the 2DST now represents a more practical alternative to the one-dimensional method.

Before implementing the 2DST, each granule of brightness temperature perturbations is interpolated onto a regularly spaced grid with approximately 17.7 and $20.3 \mathrm{~km}$ separating adjacent pixels in the along-track and cross-track directions respectively. In the centre of the AIRS swath, the resolution of this regularly spaced grid closely matches the spatial resolution of AIRS, so very little if any information is lost. Towards the edge of the swath, this grid is finer than the spatial resolution than AIRS, but the grid points will not exactly match the location of the AIRS footprints. A useful graphic of typical AIRS footprints can be found in Hoffmann et al. (2014, their Fig. 2).

As a result of using DFT algorithms, the maximum numbers of permitted wavenumber voices available in the alongtrack and cross-track directions are limited to $N_{\mathrm{AT}}-1$ and $N_{\mathrm{XT}}-1$, where $N_{\mathrm{AT}}$ and $N_{\mathrm{XT}}$ are the number of pixels in the along-track and cross-track directions (135 and 90 respectively). These wavenumber voices have corresponding wavelengths that are integer fractions of the total along-track and cross-track dimensions of the granule. Here, we compute the 2DST for wavelengths greater than around $40 \mathrm{~km}$. This is just over twice the Nyquist-sampling distance between AIRS pixels after interpolation onto our regular grid. The zeroth frequencies are omitted. Increased along-track spectral resolution at low wavenumbers can be obtained by applying the 2DST to two or more adjacent granules, thus increasing the number of along-track pixels.

\subsection{AIRS gravity wave properties measured by the 2DST}

The results of our 2DST analysis of the selected AIRS granules over South Georgia and the Antarctic Peninsula are shown in Figs. 5 and 6 respectively.
In both Figures, panel a shows the brightness temperature perturbation measurements calculated as described in Sect. 2.1. Note that the colour scale is chosen so as to make wave perturbations clearer by eye, but at some locations it is saturated.

Clear wave-like perturbations are observed in both granules directly over and to the east of the mountain ranges. As in previous work, such clear wave-like perturbations are attributable to gravity waves with a high degree of certainty.

Reconstructed 2DST temperature perturbations $T_{2-\text { DST }}^{\prime}$ are shown in panel $b$. These are found by taking the real part of the complex 2DST object $\xi\left(\tau_{x}, \tau_{y}\right)$ as described in Sect. 3.1.

The image $T^{\prime}{ }_{2-D S T}$ shows the dominant wave features in the granule reconstructed using only the pre-defined range of permitted wavenumber voices in the 2DST. Since we only consider the coefficients of the dominant wavenumber at each location, this reconstruction cannot be perfect, but it provides a visual inspection of how well the 2DST outputs represent the dominant wave characteristics of the granule. The $T_{2-\text { DST }}^{\prime}$ image can be used to "fine-tune" the 2DST by changing the windowing function, by adjusting the scaling parameter $c$ or by redefining the range of frequency voices until the desired outcome is achieved. Such fine-tuning flexibility cannot be so easily achieved using the 1DST method.

Generally, the agreement between reconstructed wave features in Fig. 5b and AIRS measurements in Fig. 5a is very good, but some uncertainties remain. As discussed in Sect. 4.3, there is some discrepancy regarding the spatial extent of some wave features, such as a small positive wave crest located just south-west of South Georgia which appears to be located slightly east, with an apparently slightly overestimated amplitude, than is observed in the AIRS measurements. Conversely, at $63^{\circ} \mathrm{S}, 28^{\circ} \mathrm{W}$, a positive bow-shaped wave crest is observed in the AIRS measurements but is underestimated in the reconstruction. As mentioned in Sect. 4.3, these small misrepresentations are not a limitation of the 2DST itself but rather the forced extraction of gravity wave parameters from the 4-D S-transform object in order to create the 2-D reconstruction, where only one single wave feature with the largest localised spectral amplitude is assigned at each location. The overall agreement is still very good, but future work to improve the extraction of gravity wave parameters from the 4-D S-transform object may help to resolve some of these discrepancies.

Figures $5 \mathrm{c}$ and $6 \mathrm{c}$ show full underlying wave amplitudes $\left|T^{\prime}\right|_{2-\text { DST }}$ for each granule. This is found by taking the absolute magnitude of the complex 2DST object $\xi\left(\tau_{x}, \tau_{y}\right)$ as described in Sect. 3.1. This property provides us with a useful metric with which to define regions of the granule which do or do not contain wave-like perturbations, such that we can limit spurious detections (e.g. McDonald, 2012). In Figs. 5h and $6 \mathrm{c}-\mathrm{h}$, we exclude regions of each granule where the underlying wave amplitude is more than 1 standard deviation below the mean underlying wave amplitude of the granule. 

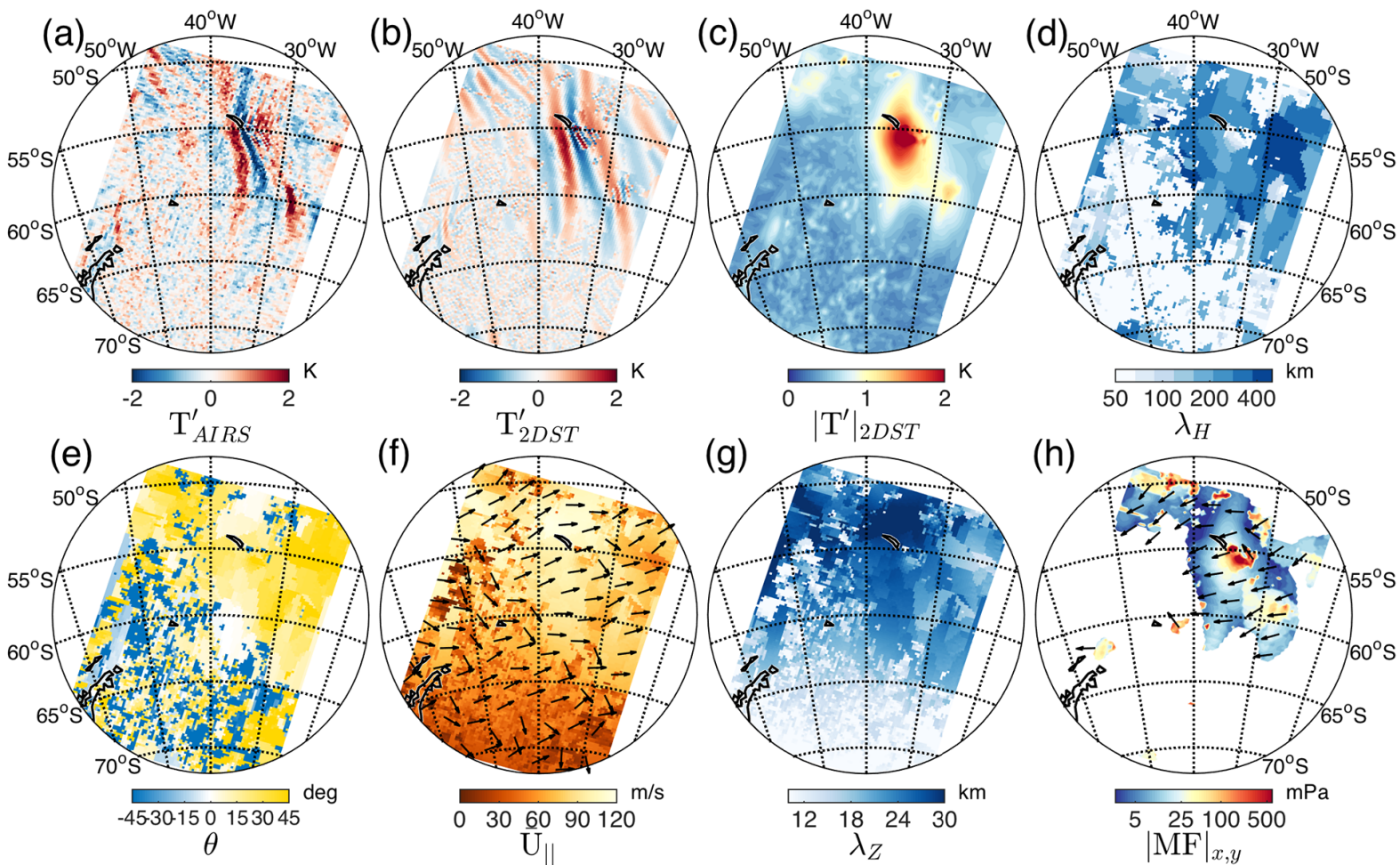

Figure 5. Orthographic projections of a granule of AIRS brightness temperature measurements $T_{\text {AIRS }}^{\prime}$ (a) over South Georgia at around 03:00 UTC on 6 September 2003 and selected outputs (b-e) of our 2DST analysis using the Elliptic-Bessel window. This granule was also analysed by Alexander et al. (2009) (their Fig. 3) using a one-dimensional S-transform method. The 2DST outputs shown here are reconstructed brightness temperature perturbations $T_{2-\mathrm{DST}}^{\prime}(\mathbf{b})$, underlying wave amplitudes $\left|T^{\prime}\right|_{2-\mathrm{DST}}$ (c), horizontal wavelengths $\lambda_{\mathrm{H}}(\mathbf{d})$ and wave propagation directions $\theta$ (e) in degrees anticlockwise from the east. Also shown are mean wind speed parallel to the horizontal wavenumber vectors $\bar{U}_{\|}$(f) from ECMWF operational analyses at $z \approx 40 \mathrm{~km}$, vertical wavelengths $\lambda_{Z}$ (g) and the magnitude of the horizontal component of vertical momentum flux $|\mathrm{MF}|_{x, y}(\mathbf{h})$. Black arrows in (f) and (h) show the horizontal direction of $\bar{U}_{||}$and $|\mathrm{MF}|_{x, y}$ respectively. For details, see text.

In Fig. 5c-g, we do not exclude such regions for discussion purposes, so as to provide an example of the data we would otherwise omit.

Figures $5 \mathrm{~d}$ and $6 \mathrm{~d}$ show absolute horizontal wavelengths $\lambda_{\mathrm{H}}=2 \pi\left(k_{\mathrm{AT}}^{2}+k_{\mathrm{XT}}^{2}\right)^{-1 / 2}$, where $k_{\mathrm{AT}}$ and $k_{\mathrm{XT}}$ are the alongtrack and cross-track angular wavenumbers respectively. We can see that these horizontal wavelengths clearly define different regimes of the dominant wave features of the granules, as in the test case in Sect. 3.1, though the AIRS data are more complex. In the South Georgia granule in Fig. 5d, we see that the island lies within a wave field where long horizontal wavelengths are dominant around and to the east of the island over the ocean, with their wavenumber vectors aligned roughly parallel to the direction of the mean flow. This is characteristic of a wing-shaped mountain wave field (Alexander and Grimsdell, 2013) and is in good agreement with visual inspection of the granule itself.

In Figs. 5e and 6e, we show the orientation of the horizontal wavenumber vector measured anticlockwise from east. $\theta$ is calculated by first projecting the along-track and crosstrack wavenumber vectors $k_{x}$ and $k_{y}$ into their zonal and meridional components $k$ and $l$ using the azimuths of the along-track and cross-track directions at each location on the granule, then taking $\theta=\tan ^{-1}\left(\frac{l}{k}\right)$. Note that $\theta$ only describes the orientation and not the true horizontal direction of propagation of the wavenumber vectors, which retain a $\pm 180^{\circ}$ ambiguity that we break below.

In the South Georgia granule (Fig. 5), we see that our 2DST measurements in the southern region of the granule are largely dominated by small-scale, low-amplitude, short horizontal wavelength features with random directions of propagation. Most of these features are likely to be due to noise and not attributable to coherent wave structures. By using a threshold amplitude, such regions are effectively removed, leaving well-defined regions with clear wavelike perturbations. The contribution of small-scale features that remain after this step is discussed further in Sect. 5.4. 

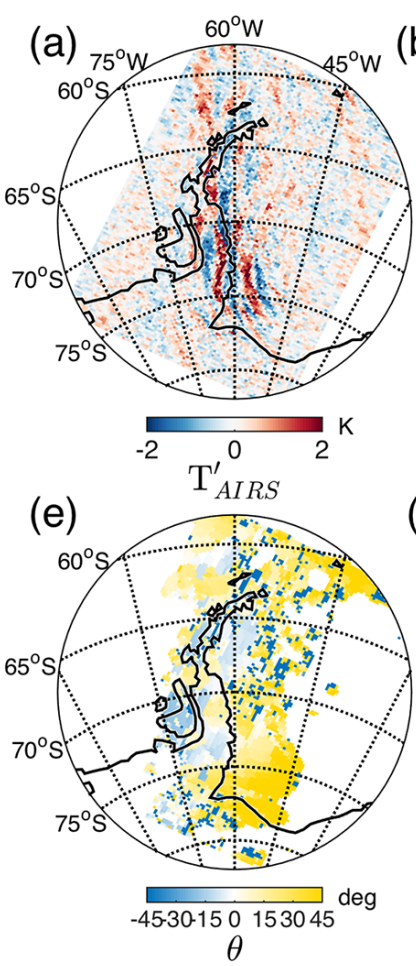

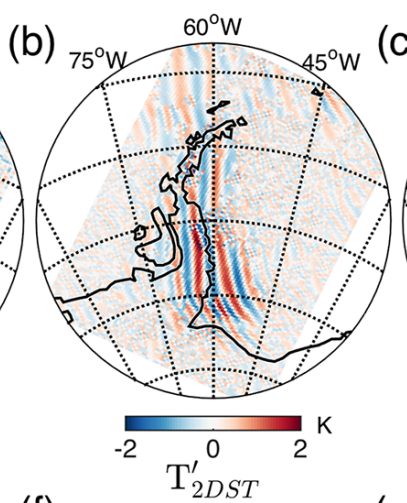

(f)

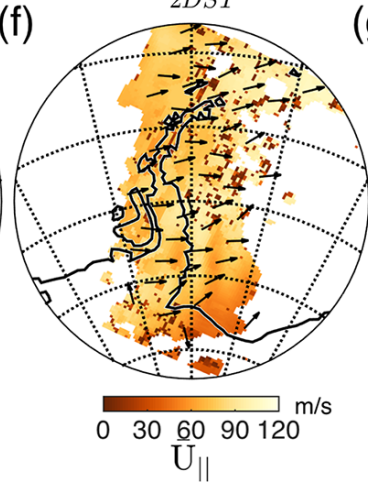

(c)

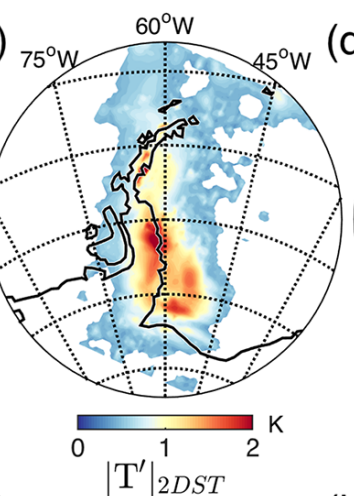

(d)

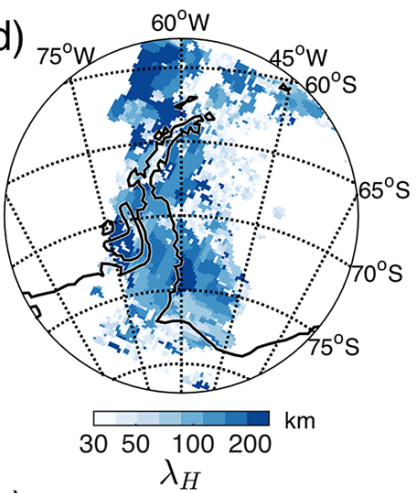

(g)

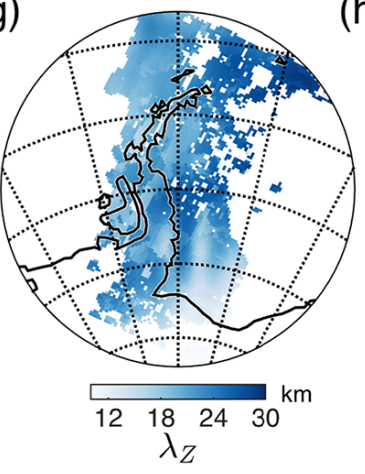

(h)

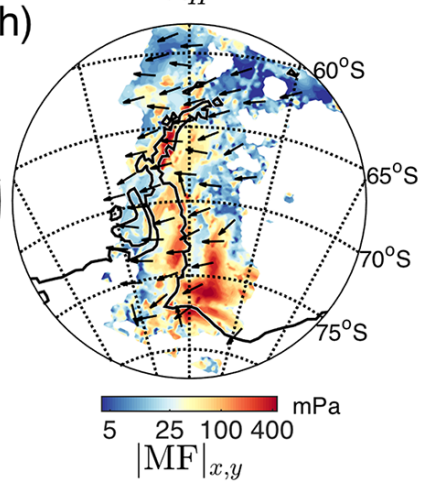

Figure 6. As Fig. 5 but for AIRS measurements over the Antarctic Peninsula around 04:00 UTC on 2 August 2010. These measurements were also analysed by Hoffmann et al. (2014, their Fig. 8) using a one-dimensional S-transform method. Here, regions in (c-h) where the underlying wave amplitude $\left|T^{\prime}\right|_{2-D S T}$ is less than 1 standard deviation below the mean are coloured white.

\subsection{Momentum fluxes}

Here we make estimates of gravity wave momentum flux for the dominant wave-like features measured by the 2DST in our selected granules, following the method of Alexander et al. (2009).

Ern et al. (2004) showed that the zonal and meridional components of gravity wave momentum flux $\mathrm{MF}_{x}$ and $\mathrm{MF}_{y}$ can be given by

$$
\left(\mathrm{MF}_{x}, \mathrm{MF}_{y}\right)=\frac{\rho}{2}\left(\frac{g}{N}\right)^{2}\left(\frac{T_{\mathrm{a}}^{\prime}}{\bar{T}}\right)^{2}\left(\frac{k}{m}, \frac{l}{m}\right)
$$

where $\rho$ is density at a height of $40 \mathrm{~km}, g$ is the acceleration due to gravity, $N$ is the buoyancy frequency, $T_{\mathrm{a}}^{\prime}$ is the attenuation-scaled full underlying wave amplitude, $\bar{T}$ is the background temperature and $k, l$ and $m$ are wavenumbers in the zonal, meridional and vertical directions respectively. Equation (15) assumes the mid-frequency approximation (Fritts and Alexander, 2003, their Sect. 2.1), which is valid for a large portion of the observable gravity wave spectrum. For these waves, $m^{2} \ll\left(k^{2}+l^{2}\right)$ and Coriolis effects are negligible, as is the case for nearly all waves measured here due to the size of the AIRS beam footprint (Hoffmann et al., 2014) and the vertical weighting function of the $667.77 \mathrm{~cm}^{-1}$ channel (Alexander and Barnet, 2007). When compared with momentum fluxes calculated without mak- ing the mid-frequency approximation, Ern et al. (2004) found discrepancies typically not exceeding around $10 \%$.

We also have a $\pm 180^{\circ}$ ambiguity in direction of propagation, which we break by assuming the waves in our granules always propagate against the mean flow. One last variable in Eq. (15) is vertical wavenumber $m=2 \pi / \lambda_{Z}$. Under the assumption that the waves are upwardly propagating mountain waves with ground-based phase velocity equal to $0, \lambda_{Z}$ is given as

$\lambda_{Z} \approx \frac{2 \pi \bar{U}_{\|}}{N}$,

where $\bar{U}_{\|}$is the component of the mean wind speed parallel to the wave's horizontal wavenumber vector (Eckermann and Preusse, 1999). $\bar{U}_{\|}$is found by projecting the mean wind vector $\bar{U}$ in the direction of the wave propagation angle $\theta$ shown in Figs. 5e and 6e.

Figures $5 \mathrm{f}$ and $6 \mathrm{f}$ show $\bar{U}_{\|}$coincident with each granule at an altitude of $40 \mathrm{~km}$ from ECMWF operational analyses, projected onto each granule's regular grid. Orange contours and black arrows show the magnitude and direction of $\bar{U}_{\|}$respectively. Vertical wavelength $\lambda_{Z}$ is shown in Figs. $5 \mathrm{~g}$ and $6 \mathrm{~g}$.

Towards the south-eastern corner of both granules, mean wind speeds become quite weak. As they fall below around $40 \mathrm{~m} \mathrm{~s}^{-1}$, vertical wavelengths start to drop below the vertical resolution limit of the AIRS channel. The wave field 
may continue into this region, but the vertical wavelengths may be too short to be resolved such that wave amplitudes are attenuated to below the ambient noise level. This is particularly clear in the Antarctic Peninsula granule, where detectable wave fronts abruptly terminate just as $\bar{U}_{\|}$begins to fall below $30 \mathrm{~m} \mathrm{~s}^{-1}$.

No further wave amplitude attenuation corrections were applied to regions where vertical wavelengths are inferred to be below $12 \mathrm{~km}$, since this is beyond the resolution limit of the weighting function of the $667.77 \mathrm{~cm}^{-1}$ channel. It should be noted, however, that although theoretically valid, the attenuation correction applied here can in some cases increase wave amplitudes by perhaps an order of magnitude or more, despite being significantly susceptible to errors in wind speed, wave direction or both. This correction should be applied with caution, and the effects of its inclusion are discussed below and in Sect. 5.4.

In the South Georgia granule, peak momentum flux values of more than $500 \mathrm{mPa}$ are associated with a small region of large amplitude and short horizontal wavelength wave features, located toward the south-eastern tip of the island. In the Antarctic Peninsula granule, momentum fluxes of a few hundred millipascals are generally co-located with the clearly visible wave structures in the raw brightness temperature perturbations just downwind of the peninsula.

The key strength of the results presented here is the muchimproved spatial-spectral localisation and resolution capabilities provided by full two-dimensional treatment of the AIRS data. Confidence in the accuracy of subsequent measured quantities in our 2DST-based analysis is thus greatly improved over previous 1-D S-transform-based methods. Understandably, the former is more computationally intensive than the latter, and this should be considered if data sets are large or computational resources are limited.

Although the magnitude, direction and distribution of momentum fluxes in both granules are broadly in line with previous AIRS gravity wave studies in the region (e.g Alexander and Teitelbaum, 2007; Alexander et al., 2009; Alexander and Teitelbaum, 2011), momentum flux magnitudes are much higher than those observed in studies using limb-sounder data sets (e.g. Ern et al., 2004, 2011; Alexander et al., 2008; Geller et al., 2013; Hindley et al., 2015; Wright et al., 2016a), which generally do not exceed a few tens of $\mathrm{mPa}$ in this region. While we would not expect the magnitude of these fluxes to be the same as those observed by limb sounders (firstly, nadir-sounding instruments are generally more sensitive to waves with longer vertical wavelengths and hence higher momentum fluxes due to the deep vertical weighting function (Alexander and Barnet, 2007); secondly, momentum flux estimates from limb sounders are typically lowerbound estimates due to the projection of horizontal wavelengths (Ern et al., 2004); and, thirdly, wavelength-dependent wave amplitude attenuation corrections are not generally applied to limb-sounder results), we note that our results and those of other AIRS gravity wave studies in this region, which use a correction factor for wave amplitude attenuation based upon a vertical wavelength estimation, are substantially higher. This effect and its implications are discussed in the next section.

\subsection{Small-scale perturbations and the attenuation correction}

In the implementation of any spectral image processing, it is important to strike a balance between accurate measurement of the desired properties and the spurious interpretation of noise. One of the advantages of AIRS measurements is the high horizontal resolution of the data. With the close exception of the Infrared Atmospheric Sounding Interferometer (IASI; e.g. Clerbaux et al., 2009), currently no other spaceborne instrument can measure stratospheric gravity waves with comparable horizontal resolution to AIRS. Therefore, accurate measurement of resolved waves with horizontal wavelengths close to the AIRS resolution limit are of great importance. Such short horizontal wavelength waves, if reliably resolved, will generally carry higher momentum fluxes via Eq. (15). However, as we approach the resolution limits of AIRS measurements and our spectral methods, our confidence in the accuracy of our measurement of such waves decreases.

In Fig. 5, our 2DST analysis resolves a very small region of short horizontal wavelengths over the south-eastern tip of South Georgia. Wave perturbations in this small region are just a few pixels across, but their uncorrected brightness temperature perturbations are large, with peaks of order 5-6 K. If these perturbations were located almost anywhere else on the granule we would likely attribute them to retrieval noise.

However, gravity waves with very short horizontal wavelengths, tightly packed in a region immediately downwind of a mountainous island, are in good agreement with mountain wave theory. Examples of such waves can be found in, for example, the modelling studies of Shutts and Vosper (2011) and Alexander and Teitelbaum (2011). If these waves are indeed real, their accurate measurement is of great importance.

An added complication is introduced as a result of the mountain wave assumption used. Since these waves are only a few pixels across, their directions of propagation are difficult to define, introducing a random element. The component of the mean wind parallel to the horizontal wavenumber vector can thus be very low, which decreases the vertical wavelength estimate, which in turn increases the attenuation correction applied to the observed temperature perturbations. This attenuation correction can increase temperature perturbations by $400 \%$ or more which, since momentum flux is proportional to the square of wave amplitude, can increase our estimate of momentum flux to extremely large values.

Thus, by applying a correction factor for wave amplitude attenuation, very small-scale low-confidence perturbations can yield extremely high momentum fluxes which can dominate the momentum budget of the entire granule if the moun- 

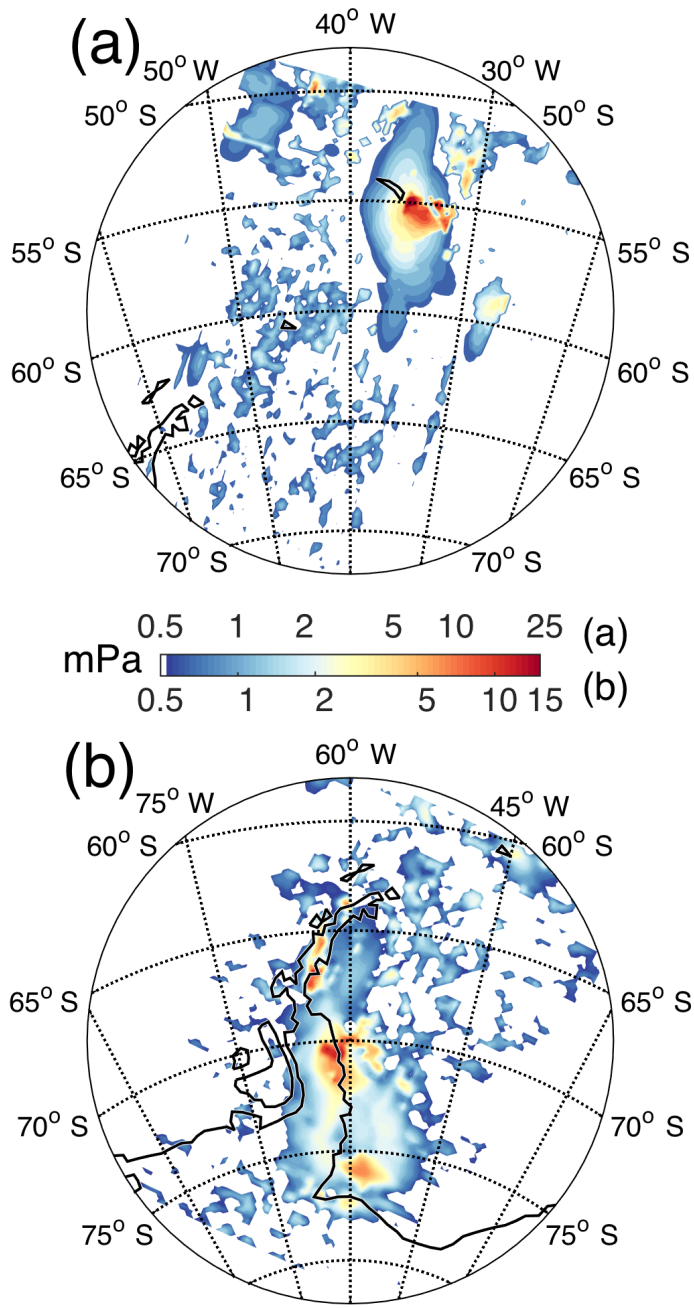

Figure 7. As Figs. 5h and $6 \mathrm{~h}$ but for estimates of the magnitude of gravity wave momentum flux over South Georgia (a) and the Antarctic Peninsula (b) calculated without correcting for attenuation of wave amplitude as discussed in Sect. 5. Momentum fluxes less than $0.5 \mathrm{mPa}$ are coloured white.

tain wave assumption is used, correctly or otherwise. This is evident in the South Georgia granule studied by Alexander et al. (2009) and shown in our Fig. 5, where peak momentum fluxes are localised over only a few large-amplitude pixels just over the south-eastern tip of the island. In Fig. 5h, the flux peaks at almost $1000 \mathrm{mPa}$ for one pixel in this small region, though the colour scale is saturated. Whether these fluxes are real or not, they can nevertheless be extremely large and should be approached with caution.

Figure $7 \mathrm{a}$ and $\mathrm{b}$ show gravity wave momentum fluxes over the South Georgia and Antarctic Peninsula granules as shown in Figs. 5 and 6, only with fluxes calculated without scaling the temperature perturbations for attenuation as described in Sect. 5. The highest momentum flux values in Fig. 7a and b are around 70 and $30 \mathrm{mPa}$ respectively, located within small regions in the wave field where the colour scale is saturated.
As is expected, these values are much lower than those calculated using the attenuation-corrected wave amplitudes.

If these extremely high fluxes correspond to real waves, then their measurement is of crucial importance. However, if such perturbations are simply instrument noise and their fluxes are spurious, then the biases and errors introduced by their inclusion in broader studies could be very large.

Without a priori knowledge of the wave environment, which is most readily gained by visual inspection of the AIRS measurements, it would be unwise to include the fluxes from these small-scale perturbations in any automated analysis. Indeed, Wright et al. (2015) suggest that when AIRS granules are pre-smoothed with a boxcar of width 3 pixels, resolved momentum fluxes calculated using the method of Alexander et al. (2009) can be reduced by a order of magnitude. This suggests that these small-scale, high-momentum flux features are reasonably common and can impact largerscale momentum flux estimates, either realistically or spuriously.

Further work investigating this problem is encouraged. Pre-smoothing granules so as to exclude these perturbations (Wright et al., 2015, 2016b) is one solution. Excluding even more wavenumber voices corresponding to short horizontal wavelengths from our 2DST analysis is another solution. However, in cases such as the South Georgia granule presented in Fig. 5, where such perturbations may well be physical, this exclusion can reduce peak momentum fluxes by orders of magnitude, introducing a systematic low bias and thus further uncertainty.

\section{Summary and conclusions}

In this study, we have applied the 2DST to granules of AIRS measurements, extracting gravity wave amplitudes, wavelengths and directions of propagation. Our 2DST method builds upon the work of Alexander and Barnet (2007), who used the 1-D Stockwell transform for the same purpose. Their method can introduce a strong cross-track bias problem, which we solve by using a full 2DST.

We first define our 2DST implementation and test it on a specified wave field containing synthetic waves with known amplitudes, wavelengths and directions of propagation. We find that the 2DST provides very good spatial representation of the dominant spectral components of the specified wave field, accurately measuring wavelengths and orientations of all the synthetic waves.

Due to the spread of spectral power in the spectral domain and wave undersampling in the spatial domain, we find that localised wave amplitudes as measured by the 2DST are reduced by more than a factor of two when the typical Gaussian windowing function is used in the Stockwell transform. We compensate for this by decreasing the scaling parameter $c$ and by replacing the Gaussian window with a new alternative Elliptic-Bessel window, which we test on a granule of 
AIRS measurements over the southern Andes. We find that this new spectral window provides a better balance between spatial-spectral localisation and the accurate measurement of wave amplitudes. Wave amplitude recovery is thus improved to around 80 to $90 \%$ of input values. We also demonstrate that this new window is indeed valid for use in the 2DST by showing its spatial integral is equal to unity.

Next, we measure gravity wave amplitudes, horizontal wavelengths and directions of propagation in two granules of AIRS measurements over South Georgia and the Drake Passage/Antarctic Peninsula region. Our 2DST method significantly improves two-dimensional representation of the dominant spectral features of the granules over previous 1DST methods. These spectral features are directly measured in both dimensions simultaneously for each location of the granule, without the introduction of potential biases caused by the use of averaged co-spectra. This is a clear advantage over previous methods.
Another key advantage of our 2DST method is the ability to visually inspect the quality of our spectral analysis. By taking the real parts of the dominant localised spectral coefficients at each location, a reconstruction of the granule can be created. This can be used to fine-tune the adjustable parameters and provide a useful sanity check on the performance of the 2DST. Future work may involve comparing this output to the original data via a variance argument or similar, such that we can obtain a quantitative measure of the quality of the 2DST analysis for quality control purposes in largerscale studies.

To conclude, our new 2DST-based gravity wave analysis method for AIRS data makes significant improvements over current methods in several key areas, and we would advocate its use in future work. 
Appendix A: Admissibility of the Elliptic-Bessel window in the Stockwell transform

In Sect. 4 we introduced the Elliptic-Bessel window as a new apodizing function for the 2DST. One requirement for any apodizing function for use in the Stockwell transform is that its spatial sum must be equal to unity. If this condition is satisfied, the spatial sum of the 2DST is equal to the 2-D Fourier transform, making the 2DST fully invertible.

In this appendix we demonstrate that the Elliptic-Bessel window is admissible as an apodizing function in the Stransform. To do this, we must first find the spatial analogue of the wavenumber-domain ellipse we defined in Eq. (12). We must then take the spatial integral of this function to demonstrate that it is equal to unity.

\section{A1 The Elliptic-Bessel window in the spatial domain}

The Elliptic-Bessel window is defined in the wavenumber $\left(\alpha_{x}, \alpha_{y}\right)$ domain as

$W_{\mathrm{eb}}\left(\alpha_{x}, \alpha_{y}, k_{x}, k_{y}\right)=\left\{\begin{array}{l}0 \text { for }\left(\frac{\alpha_{x}}{a}\right)^{2}+\left(\frac{\alpha_{y}}{b}\right)^{2} \geqslant 1 \\ 1 \text { for }\left(\frac{\alpha_{x}}{a}\right)^{2}+\left(\frac{\alpha_{y}}{b}\right)^{2}<1\end{array}\right.$

where $a=\left|k_{x}\right| / 2 \pi c$ and $b=\left|k_{y}\right| / 2 \pi c$ are the half-widths of the ellipse in the $\alpha_{x}$ and $\alpha_{y}$ directions (see Fig. 3b). The spatial-domain form of the Elliptic-Bessel window, denoted here by $w_{\mathrm{eb}}\left(x, y, k_{x}, k_{y}\right)$, is found by taking the inverse 2-D Fourier transform of Eq. (A1) as

$$
\begin{aligned}
& w_{\mathrm{eb}}\left(x, y, k_{x}, k_{y}\right) \\
& =\mathfrak{F}_{x}^{-1} \mathfrak{F}_{y}^{-1}\left[W_{\mathrm{eb}}\left(\alpha_{x}, \alpha_{y}, k_{x}, k_{y}\right)\right] \\
& =\frac{1}{4 \pi^{2}} \int_{\infty}^{\infty} \int_{\infty}^{\infty} W_{\mathrm{eb}}\left(\alpha_{x}, \alpha_{y}, k_{x}, k_{y}\right) e^{i\left(\alpha_{x} x+\alpha_{y} y\right)} \mathrm{d} \alpha_{x} \mathrm{~d} \alpha_{y} .
\end{aligned}
$$

Since $W_{\mathrm{eb}}\left(\alpha_{x}, \alpha_{y}, k_{x}, k_{y}\right)=1$ within the ellipse and 0 everywhere else, and has double symmetry, we can change the limits of integration to be the boundaries of the ellipse, expressing the total integral as a sum of four equal quadrants:

$$
\begin{aligned}
& w_{\mathrm{eb}}\left(x, y, k_{x}, k_{y}\right)= \\
& \frac{4}{4 \pi^{2}} \int_{0}^{\sqrt{b^{2}-\frac{\alpha_{x}^{2} b^{2}}{a^{2}}}} \int_{0}^{a} e^{i\left(\alpha_{x} x+\alpha_{y} y\right)} \mathrm{d} \alpha_{x} \mathrm{~d} \alpha_{y} .
\end{aligned}
$$

We then recognise that the exponential term in the transform above can be replaced with sine and cosine functions as

$$
\begin{aligned}
& e^{i\left(\alpha_{x} x+\alpha_{y} y\right)} \\
= & \left(\cos \left(\alpha_{x} x\right)+i \sin \left(\alpha_{x} x\right)\right)\left(\cos \left(\alpha_{y} y\right)+i \sin \left(\alpha_{y} y\right)\right) \\
= & \cos \left(\alpha_{x} x\right) \cos \left(\alpha_{y} y\right)+i \sin \left(\alpha_{x} x\right) \cos \left(\alpha_{y} y\right) \\
& +i \sin \left(\alpha_{y} y\right) \cos \left(\alpha_{x} x\right)-\sin \left(\alpha_{x} x\right) \sin \left(\alpha_{y} y\right) .
\end{aligned}
$$

We can omit the last three $\sin ()$ terms in Eq. (A4) since, due to the symmetry of the sine function around $(0,0)$, each term will eventually sum to 0 . We can then rewrite Eq. (A3) as

$$
\begin{aligned}
& w_{\mathrm{eb}}\left(x, y, k_{x}, k_{y}\right)= \\
& \frac{4}{4 \pi^{2}} \int_{0}^{\sqrt{b^{2}-\frac{\alpha_{x}^{2} b^{2}}{a^{2}}}} \int_{0}^{a} \cos \left(\alpha_{x} x\right) \cos \left(\alpha_{y} y\right) \mathrm{d} \alpha_{x} \mathrm{~d} \alpha_{y} .
\end{aligned}
$$

This integral can be further simplified if we switch to polar coordinates using the substitutions $\alpha_{x}=\operatorname{ar} \cos (\phi)$ and $\alpha_{y}=$ $b r \sin (\phi)$ after which the expression in Eq. (A5) becomes

$w_{\mathrm{eb}}\left(x, y, k_{x}, k_{y}\right)=$

$\frac{a b}{4 \pi^{2}} \int_{0}^{2 \pi} \int_{0}^{1} \cos (\operatorname{ar} x \cos (\phi)) \cos (b r y \sin (\phi)) r \mathrm{~d} r \mathrm{~d} \phi$.

Next we substitute $\mathcal{A}=\operatorname{ar} x$ and $\mathcal{B}=$ bry and, using multiple angle formulae, rewrite Eq. (A6) as

$$
\begin{aligned}
& w_{\mathrm{eb}}\left(x, y, k_{x}, k_{y}\right) \\
= & \frac{a b}{4 \pi^{2}} \int_{0}^{1} r \int_{0}^{2 \pi} \cos (\mathcal{A} \cos (\phi)) \cos (\mathcal{B} \sin (\phi)) \mathrm{d} \phi \mathrm{d} r \\
= & \frac{a b}{8 \pi^{2}} \int_{0}^{1} r \int_{0}^{2 \pi}[\cos (\mathcal{A} \cos \phi+\mathcal{B} \sin \phi) \\
& +\cos (\mathcal{A} \cos \phi-\mathcal{B} \sin \phi)] \mathrm{d} \phi \mathrm{d} r \\
= & \frac{a b}{8 \pi^{2}} \int_{0}^{1} r \int_{0}^{2 \pi}\left[\operatorname { c o s } \left(\sqrt{\mathcal{A}^{2}+\mathcal{B}^{2}} \cos (\phi-\Lambda)\right.\right. \\
& +\cos \left(\sqrt{\mathcal{A}^{2}+\mathcal{B}^{2}} \cos (\phi+\Lambda)\right] \mathrm{d} \phi \mathrm{d} r,
\end{aligned}
$$

where $\Lambda=\tan ^{-1}(\mathcal{B} / \mathcal{A})$. Here, $\Lambda$ is simply an arbitrary phase due to the periodicity of the cosine function when integrated over 0 to $2 \pi$, so the integrals of both terms in the square brackets in Eq. (A7) will be equal. Hence we can sim- 
ply add these terms such that we have

$$
\begin{aligned}
& w_{\mathrm{eb}}\left(x, y, k_{x}, k_{y}\right)= \\
& \frac{a b}{8 \pi^{2}} \int_{0}^{1} r \int_{0}^{2 \pi} 2 \cos \left(\sqrt{\mathcal{A}^{2}+\mathcal{B}^{2}} \cos \phi\right) \mathrm{d} \phi \mathrm{d} r .
\end{aligned}
$$

Next we recall the integral definition of the zeroth-order Bessel function of the first kind $\mathbf{J}_{0}(x)$ (Abramowitz and Stegun, 1964) given as

$\mathbf{J}_{0}(x)=\frac{1}{2 \pi} \int_{0}^{2 \pi} \cos (x \cos \phi) \mathrm{d} \phi$

and substitute into Eq. (A8) and reintroduce our substitutions of $\mathcal{A}=$ ar $x$ and $\mathcal{B}=$ bry to give

$w_{\mathrm{eb}}\left(x, y, k_{x}, k_{y}\right)=\frac{a b}{2 \pi} \int_{0}^{1} \mathbf{J}_{0}\left(r \sqrt{a^{2} x^{2}+b^{2} y^{2}}\right) r \mathrm{~d} r$.

We now use a new substitution that $\xi=r \sqrt{a^{2} x^{2}+b^{2} y^{2}}$ and rewrite Eq. (A10) as

$w_{\mathrm{eb}}\left(x, y, k_{x}, k_{y}\right)=\frac{a b}{2 \pi} \int_{0}^{\sqrt{a^{2} x^{2}+b^{2} y^{2}}} \frac{\xi \mathbf{J}_{0}(\xi)}{a^{2} x^{2}+b^{2} y^{2}} \mathrm{~d} \xi$.

Next we use the standard result (e.g. Abramowitz and Stegun, 1964) that

$\int_{x_{1}}^{x_{2}} x \mathbf{J}_{0}(x) \mathrm{d} x=\left.x \mathbf{J}_{1}(x)\right|_{x_{1}} ^{x_{2}}$

to rewrite Eq. (A11) as

$$
\begin{aligned}
w_{\mathrm{eb}}\left(x, y, k_{x}, k_{y}\right) & =\left.\frac{a b}{2 \pi} \frac{\xi \mathbf{J}_{1}(\xi)}{\left(a^{2} x^{2}+b^{2} y^{2}\right)}\right|_{0} ^{\sqrt{a^{2} x^{2}+b^{2} y^{2}}} \\
& =\frac{a b}{2 \pi} \frac{\mathbf{J}_{1}\left(\sqrt{a^{2} x^{2}+b^{2} y^{2}}\right)}{\sqrt{a^{2} x^{2}+b^{2} y^{2}}} .
\end{aligned}
$$

Finally, recalling that $a=\left|k_{x}\right| / 2 \pi c$ and $b=\left|k_{y}\right| / 2 \pi c$ are the half-widths of the original ellipse in Eq. (A1), we now can write the analytical expression for the spatial form of the Elliptic-Bessel window as

$w_{\mathrm{eb}}\left(x, y, k_{x}, k_{y}\right)=\frac{\left|k_{x}\right|\left|k_{y}\right|}{8 \pi^{3} c^{2}} \frac{\mathbf{J}_{1}(z)}{z}$,

where

$z=\frac{1}{2 \pi c} \sqrt{k_{x}^{2} x^{2}+k_{y}^{2} y^{2}}$.
This spatial-domain form of the Elliptic-Bessel window in Eq. (A14) is plotted in Fig. 3d.

Equation (A14) describes a $\mathbf{J}_{1}(z)$ function within a scaled $1 / z$ envelope. Because of this, the terms involving $z$ in Eq. (A14) converge to $1 / 2$ as $x \rightarrow 0$ and $y \rightarrow 0$, such that the central region of the function has peak value of $\left|k_{x}\right|\left|k_{y}\right| / 16 \pi^{3} c^{2}$, as shown in Fig. 3d. Interestingly, this value is equal to half the peak value of the equivalent Gaussian window shown in Fig. 3c. The central peak of the Elliptic-Bessel window is also, for each frequency voice, slightly broader than that of the equivalent voice Gaussian.

\section{A2 Spatial integral of the Elliptic-Bessel window}

Now that we have found an analytical expression for the spatial-domain form of the Elliptic-Bessel window (Eq. A14), we can proceed to check that it is admissible as an apodizing function in the 2-D Stockwell transform namely that its spatial sum is equal to unity (e.g. Pinnegar and Mansinha, 2003). The spatial sum of Eq. (A14), denoted here by $\mathbb{I}$, can be written as

$\mathbb{I}=\int_{-\infty}^{\infty} \int_{-\infty}^{\infty} \frac{\left|k_{x}\right|\left|k_{y}\right|}{8 \pi^{3} c^{2}} \frac{\mathbf{J}_{1}(z)}{z} \mathrm{~d} x \mathrm{~d} y$.

This integral can be simplified if we reintroduce our substitutions $a=\left|k_{x}\right| / 2 \pi c$ and $b=\left|k_{y}\right| / 2 \pi c$ and switch to polar coordinates, using the substitutions $x=\frac{\Lambda \cos (\varphi)}{a}$ and $y=\frac{\Lambda \sin (\varphi)}{b}$ to give

$$
\begin{aligned}
\mathbb{I} & =\frac{a b}{2 \pi} \int_{0}^{2 \pi} \int_{0}^{\infty} \frac{\mathbf{J}_{1}(\Lambda)}{\Lambda} \frac{\Lambda}{a b} \mathrm{~d} \Lambda \mathrm{d} \varphi \\
& =\frac{1}{2 \pi} \int_{0}^{2 \pi} \int_{0}^{\infty} \mathbf{J}_{1}(\Lambda) \mathrm{d} \Lambda \mathrm{d} \varphi \\
& =\frac{2 \pi}{2 \pi} \int_{0}^{\infty} \mathbf{J}_{1}(\Lambda) \mathrm{d} \Lambda .
\end{aligned}
$$

Using the standard result (e.g. Abramowitz and Stegun, 1964) that

$\int_{x_{1}}^{x_{2}} \mathbf{J}_{1}(x) \mathrm{d} x=-\left.\mathbf{J}_{0}(x)\right|_{x_{1}} ^{x_{2}}$,

we see that Eq. (A16) becomes

$$
\begin{aligned}
\mathbb{I} & =-\left.\mathbf{J}_{0}(\Lambda)\right|_{0} ^{\infty} \\
& =(0)-(-1) \\
& =1
\end{aligned}
$$

as required. This result confirms that the spatial sum of $w_{\mathrm{eb}}\left(x, y, k_{x}, k_{y}\right)$ is indeed equal to unity; thus the Elliptic- 
Bessel window is admissible as an apodizing window for the 2-D Stockwell transform.

\section{A3 Admissibility of other windows}

In this appendix so far, we have found a useful analytical expression for spatial form of the Elliptic-Bessel window presented in this study. We have then shown that its spatial integral is equal to unity and it is thus admissible as an apodizing function in the 2-D Stockwell transform. In other cases, a quick test may be performed on candidate S-transform windowing functions to check whether their spatial integral is unity.

If we take the spatial integral $\mathbb{I}$ of the spatial-domain form of a candidate windowing function $w\left(x, y, k_{x}, k_{y}\right)$, namely

$\mathbb{I}=\int_{-\infty}^{\infty} \int_{-\infty}^{\infty} w\left(x, y, k_{x}, k_{y}\right) \mathrm{d} x \mathrm{~d} y$,

and introduce the factor $e^{-i\left(k_{x} x+k_{y} y\right)}$, noting that when $k_{x}=$ $k_{y}=0$ this factor is equal to unity, then $\mathbb{I}$ can be written as

$$
\begin{aligned}
\mathbb{I} & =\left.\int_{-\infty}^{\infty} \int_{-\infty}^{\infty} w\left(x, y, k_{x}, k_{y}\right) e^{-i\left(k_{x} x+k_{y} y\right)} \mathrm{d} x \mathrm{~d} y\right|_{k_{x}=k_{y}=0} \\
& =\left.W\left(\alpha_{x}, \alpha_{y}, k_{x}, k_{y}\right)\right|_{k_{x}=k_{y}=0}
\end{aligned}
$$

where $W\left(\alpha_{x}, \alpha_{y}, k_{x}, k_{y}\right)$ is the wavenumber domain form of the candidate window and the notation $\left.\right|_{k_{x}=k_{y}=0}$ denotes that the function is evaluated at $k_{x}=k_{y}=0$. This means that if the value of $W\left(\alpha_{x}, \alpha_{y}, k_{x}, k_{y}\right)$ evaluated at $k_{x}=0$ and $k_{y}=0$ is equal to unity, then its spatial integral will also be equal to unity. If it is not, then the candidate window is not admissible for use the Stockwell transform. Figure 3a and b show that both the Gaussian window and the Elliptic-Bessel window are equal to unity at $k_{x}=k_{y}=0$ and thus satisfy this requirement. This short test may be helpful in the design of proposed alternative S-transform windowing functions in the future. 
Acknowledgements. Neil P. Hindle is funded by a NERC studentship awarded to the University of Bath. Corwin J. Wright and Nicholas J. Mitchell are supported by NERC grant NE/K015117/1. The authors would like to thank the AIRS programme team for many years of hard work producing the data used here and also the handling editor and anonymous reviewers for their very helpful suggestions.

Edited by: L. Hoffmann

\section{References}

Abramowitz, M. and Stegun, I.: Handbook of Mathematical Functions: With Formulas, Graphs, and Mathematical Tables, Applied mathematics series, Dover Publications, 1964.

Alexander, M. J. and Barnet, C.: Using satellite observations to constrain parameterizations of gravity wave effects for global models, J. Atmos. Sci., 64, 1652-1665, doi:10.1175/JAS3897.1, 2007.

Alexander, M. J. and Grimsdell, A. W.: Seasonal cycle of orographic gravity wave occurrence above small islands in the Southern Hemisphere: Implications for effects on the general circulation, J. Geophys. Res., 118, 11589-11599, doi:10.1002/2013JD020526, 2013.

Alexander, M. J. and Teitelbaum, H.: Observation and analysis of a large amplitude mountain wave event over the Antarctic peninsula, J. Geophys. Res., 112, D21103, doi:10.1029/2006JD008368, 2007.

Alexander, M. J. and Teitelbaum, H.: Three-dimensional properties of Andes mountain waves observed by satellite: A case study, J. Geophys. Res., 116, D23110, doi:10.1029/2011JD016151, 2011.

Alexander, M. J., Gille, J., Cavanaugh, C., Coffey, M., Craig, C., Eden, T., Francis, G., Halvorson, C., Hannigan, J., Khosravi, R., Kinnison, D., Lee, H., Massie, S., Nardi, B., Barnett, J., Hepplewhite, C., Lambert, a., and Dean, V.: Global estimates of gravity wave momentum flux from High Resolution Dynamics Limb Sounder observations, J. Geophys. Res., 113, D15S18, doi:10.1029/2007JD008807, 2008.

Alexander, M. J., Eckermann, S. D., Broutman, D., and Ma, J.: Momentum flux estimates for South Georgia Island mountain waves in the stratosphere observed via satellite, Geophy. Res. Lett., 36, L12816, doi:10.1029/2009GL038587, 2009.

Alexander, M. J., Geller, M., McLandress, C., Polavarapu, S., Preusse, P., Sassi, F., Sato, K., Eckermann, S., Ern, M., Hertzog, A., Kawatani, Y., Pulido, M., Shaw, T. A., Sigmond, M., Vincent, R., and Watanabe, S.: Recent developments in gravity-wave effects in climate models and the global distribution of gravitywave momentum flux from observations and models, Q. J. Roy. Meteor. Soc., 136, 1103-1124, doi:10.1002/qj.637, 2010.

Aumann, H., Chahine, M., Gautier, C., Goldberg, M., Kalnay, E., McMillin, L., Revercomb, H., Rosenkranz, P., Smith, W., Staelin, D., Strow, L., and Susskind, J.: AIRS/AMSU/HSB on the Aqua mission: design, science objectives, data products, and processing systems, IEEE T. Geosci. Remote, 41, 253-264, doi:10.1109/TGRS.2002.808356, 2003.

Barry, R. L., Strother, S. C., and Gore, J. C.: Complex and magnitude-only preprocessing of $2 \mathrm{D}$ and 3D BOLD fMRI data at 7T, Magnetic Resonance in Medicine, 67, 867-871, doi:10.1002/mrm.23072, 2012.
Baumgaertner, A. J. G. and McDonald, A. J.: A gravity wave climatology for Antarctica compiled from Challenging Minisatellite Payload/Global Positioning System (CHAMP/GPS) radio occultations, J. Geophys. Res., 112, D05103, doi:10.1029/2006JD007504, 2007.

Brigham, E. O.: The Fast Fourier Transform, Prentice-Hall Inc., 1974.

Brown, R. A., Lauzon, M. L., and Frayne, R.: A General Description of Linear Time-Frequency Transforms and Formulation of a Fast, Invertible Transform That Samples the Continuous STransform Spectrum Nonredundantly, IEEE Trans. Sig. Proc., 58, 281-290, doi:10.1109/TSP.2009.2028972, 2010.

Butchart, N., Charlton-Perez, A. J., Cionni, I., et al.: Multimodel climate and variability of the stratosphere, J. Geophys. Res., 116, D05102, doi:10.1029/2010JD014995, d05102, 2011.

Clerbaux, C., Boynard, A., Clarisse, L., George, M., Hadji-Lazaro, J., Herbin, H., Hurtmans, D., Pommier, M., Razavi, A., Turquety, S., Wespes, C., and Coheur, P.-F.: Monitoring of atmospheric composition using the thermal infrared IASI/MetOp sounder, Atmos. Chem. Phys., 9, 6041-6054, doi:10.5194/acp-9-6041-2009, 2009.

Eckermann, S. D. and Preusse, P.: Global Measurements of Stratospheric Mountain Waves from Space, Science, 286, 1534-1537, doi:10.1126/science.286.5444.1534, 1999.

Ern, M., Preusse, P., Alexander, M. J., and Warner, C. D.: Absolute values of gravity wave momentum flux derived from satellite data, J. Geophys. Res., 109, D20103, doi:10.1029/2004JD004752, 2004.

Ern, M., Preusse, P., Gille, J. C., Hepplewhite, C. L., Mlynczak, M. G., Russell, J. M., and Riese, M.: Implications for atmospheric dynamics derived from global observations of gravity wave momentum flux in stratosphere and mesosphere, J. Geophys. Res., 116, D19107, doi:10.1029/2011JD015821, 2011.

Fritts, D. C. and Alexander, M. J.: Gravity wave dynamics and effects in the middle atmosphere, Rev. Geophys., 41, 1003, doi:10.1029/2001RG000106, 2003.

Fritts, D. C., Riggin, D. M., Balsley, B. B., and Stockwell, R. G.: Recent results with an MF radar at McMurdo, Antarctica: Characteristics and variability of motions near 12-hour period in the mesosphere, Geophy. Res. Lett., 25, 297-300, doi:10.1029/97GL03702, 1998.

Geller, M., Alexander, M. J., Love, P., Bacmeister, J., Ern, M., Hertzog, A., Manzini, E., Preusse, P., Sato, K., Scaife, A., and Zhou, T.: A Comparison between Gravity Wave Momentum Fluxes in Observations and Climate Models, J. Climate, 26, 6383-6405, doi:10.1175/JCLI-D-12-00545.1, 2013.

Gibson, P. C., Lamoureux, M. P., and Margrave, G. F.: Letter to the editor: Stockwell and wavelet transforms, J. Fourier Anal. Appl., 12, 713-721, doi:10.1007/s00041-006-6087-9, 2006.

Goodyear, B. G., Zhu, H. M., Brown, R. A., and Mitchell, J. R.: Removal of phase artifacts from fMRI data using a Stockwell transform filter improves brain activity detection, Magn. Reson. Med., 51, 16-21, doi:10.1002/mrm.10681, 2004.

Hertzog, A., Boccara, G., Vincent, R. A., Vial, F., and Cocquerez, P.: Estimation of gravity wave momentum flux and phase speeds from quasi-Lagrangian stratospheric balloon flights - Part II: Results from the Vorcore campaign in Antarctica., J. Atmos. Sci., 65, 3056-3070, doi:10.1175/2008JAS2710.1, 2008. 
Hertzog, A., Alexander, M. J., and Plougonven, R.: On the Intermittency of Gravity Wave Momentum Flux in the Stratosphere, J. Atmos. Sci., 69, 3433-3448, doi:10.1175/JAS-D-12-09.1, 2012.

Hindley, N. P., Wright, C. J., Smith, N. D., and Mitchell, N. J.: The southern stratospheric gravity wave hot spot: individual waves and their momentum fluxes measured by COSMIC GPS-RO, Atmos. Chem. Phys., 15, 7797-7818, doi:10.5194/acp-15-77972015, 2015.

Hoffmann, L. and Alexander, M. J.: Retrieval of stratospheric temperatures from Atmospheric Infrared Sounder radiance measurements for gravity wave studies, J. Geophys. Res., 114, D07105, doi:10.1029/2008JD011241, 2009.

Hoffmann, L., Xue, X., and Alexander, M. J.: A global view of stratospheric gravity wave hotspots located with Atmospheric Infrared Sounder observations, J. Geophys. Res., 118, 416-434, doi:10.1029/2012JD018658, 2013.

Hoffmann, L., Alexander, M. J., Clerbaux, C., Grimsdell, A. W., Meyer, C. I., Rößler, T., and Tournier, B.: Intercomparison of stratospheric gravity wave observations with AIRS and IASI, Atmos. Meas. Tech., 7, 4517-4537, doi:10.5194/amt-7-4517-2014, 2014.

Jiang, J. H., Wu, D. L., and Eckermann, S. D.: Upper Atmosphere Research Satellite (UARS) MLS observation of mountain waves over the Andes, J. Geophys. Res.-Atmos., 107, SOL 15-1-SOL 15-10 , doi:10.1029/2002JD002091, 2002.

Kocahan, O., Coskun, E., and Oezder, S.: Profile Measurement of Objects by Using Stockwell and Continuous Wavelet Transforms, 16th Signal Processing and Communications Applications Conference, Aydin, Turkey, 20-22 April 2008, 804-807, 2008.

Kuyuk, H. S.: On the use of Stockwell transform in structural dynamic analysis, Sadhana-Academy Proceedings in Engineering Sciences, 40, 295-306, doi:10.1007/s12046-014-0301-2, 2015.

Liu, Y.: Localization Operators for Two-Dimensional Stockwell Transforms, in: New Developments in Pseudo-Differential Operators, edited by: Rodino, L. and Wong, M. W., Operator Theory Advances and Applications, 189, 287-296, 2009.

Liu, Y. and Wong, M. W.: Inversion formulas for two-dimensional Stockwell transforms, in: Pseudo-Differential Operators: Partial Differential Equations and time-frequency analysis, edited by: Rodino, L., Schulze, B. W., and Wong, M. W., Fields Institute Communications, 52, 323-330, 2007.

Mansinha, L., Stockwell, R. G., and Lowe, R. P.: Pattern analysis with two-dimensional spectral localisation: Applications of two-dimensional S transforms, Physica A, 239, 286-295, doi:10.1016/S0378-4371(96)00487-6, 1997a.

Mansinha, L., Stockwell, R. G., Lowe, R. P., Eramian, M., and Schincariol, R. A.: Local S-spectrum analysis of 1-D and 2-D data, Phys. Earth Planet. In., 103, 329-336, doi:10.1016/S00319201(97)00047-2, 1997b.

McDonald, A. J.: Gravity wave occurrence statistics derived from paired COSMIC/FORMOSAT3 observations, J. Geophys. Res., 117, D15106, doi:10.1029/2011JD016715, 2012.

Pinnegar, C. R. and Mansinha, L.: The S-transform with windows of arbitrary and varying shape, Geophysics, 68, 381-385, doi:10.1190/1.1543223, 2003.

Plougonven, R., Arsac, A., Hertzog, A., Guez, L., and Vial, F.: Sensitivity study for mesoscale simulations of gravity waves above
Antarctica during Vorcore, Q. J. Roy. Meteor. Soc., 136, 13711377, doi:10.1002/qj.639, 2010.

Plougonven, R., Hertzog, A., and Guez, L.: Gravity waves over Antarctica and the Southern Ocean: consistent momentum fluxes in mesoscale simulations and stratospheric balloon observations, Q. J. Roy. Meteor. Soc., 139, 101-118, doi:10.1002/qj.1965, 2013.

Sato, K., Tateno, S., Watanabe, S., and Kawatani, Y.: Gravity Wave Characteristics in the Southern Hemisphere Revealed by a High-Resolution Middle-Atmosphere General Circulation Model., J. Atmos. Sci., 69, 1378-1396, doi:10.1175/JAS-D-110101.1, 2012.

Shutts, G. J. and Vosper, S. B.: Stratospheric gravity waves revealed in NWP model forecasts, Q. J. Roy. Meteor. Soc., 137, 303-317, doi:10.1002/qj.763, 2011.

Stockwell, R., Mansinha, L., and Lowe, R. P.: Localization of the complex spectrum: the S-Transform, IEEE Trans. Sig. Proc., 44, 998-1001, 1996.

Stockwell, R. G.: S-Transform Analysis of Gravity Wave Activity from a Small Scale Network of Airglow Imagers, PhD thesis, University of Western Ontario, 1999.

Stockwell, R. G.: A basis for efficient representation of the S-transform, Digital Signal Processing, 17, 371-393, doi:10.1016/j.dsp.2006.04.006, 2007.

Wright, C. J.: Detection Of Stratospheric Gravity Waves Using HIRDLS Data, PhD thesis, Trinity College, University of Oxford, 2010.

Wright, C. J.: A one-year seasonal analysis of martian gravity waves using MCS data, Icarus, 219, 274-282, doi:10.1016/j.icarus.2012.03.004, 2012.

Wright, C. J. and Gille, J. C.: Detecting overlapping gravity waves using the S-Transform, Geophy. Res. Lett., 40, 1850-1855, doi:10.1002/grl.50378, 2013.

Wright, C. J., Osprey, S. M., and Gille, J. C.: Global distributions of overlapping gravity waves in HIRDLS data, Atmos. Chem. Phys., 15, 8459-8477, doi:10.5194/acp-15-8459-2015, 2015.

Wright, C. J., Hindley, N. P., Moss, A. C., and Mitchell, N. J.: Multiinstrument gravity-wave measurements over Tierra del Fuego and the Drake Passage - Part 1: Potential energies and vertical wavelengths from AIRS, COSMIC, HIRDLS, MLS-Aura, SAAMER, SABER and radiosondes, Atmos. Meas. Tech., 9, 877-908, doi:10.5194/amt-9-877-2016, 2016a.

Wright, C. J., Hindley, N. P., and Mitchell, N. J.: Combining AIRS and MLS observations for three-dimensional gravity wave measurement, Geophy. Res. Lett., 43, 884-893, doi:10.1002/2015GL067233, 2015GL067233, 2016 b.

$\mathrm{Wu}$, D. L.: Mesoscale gravity wave variances from AMSUA radiances, Geophy. Res. Lett., 31, 1944-8007, doi:10.1029/2004GL019562, 112114, 2004.

Yan, A., Zhou, W., Yuan, Q., Yuan, S., Wu, Q., Zhao, X., and Wang, J.: Automatic seizure detection using Stockwell transform and boosting algorithm for long-term EEG, Epilepsy Behav., 45, 814, doi:10.1016/j.yebeh.2015.02.012, 2015. 